\title{
Setting the Scientific Stage for Esports Psychology: A Systematic
}

\section{Review}

3 Ismael Pedraza-Ramirez ${ }^{1 *}$, Lisa Musculus ${ }^{1}$, Markus Raab ${ }^{1,3}$, Sylvain

$4 \quad$ Laborde $^{1,2}$

5 'Department of Performance Psychology, Institute of Psychology, German Sport 6 University Cologne, Cologne, Germany

$7 \quad{ }^{2}$ University of Caen Normandy, UFR STAPS, EA 4260, France

$8 \quad{ }^{3}$ School of Applied Science, London South Bank University, UK

9

10

$11 *$ Corresponding author. German Sport University Cologne, Institute of Psychology,

12 Department of Performance Psychology, Am Sportpark Müngersdorf 6, 50933 Cologne,

13 Germany.

14 Email Address: i.pedraza@stud.dshs-koeln.de

15

16

17

18

19

20 


\section{Setting the Scientific Stage for Esports Psychology: A Systematic}

Review

Competitive gaming, better known as electronic sports (esports), is rapidly growing in popularity. We systematically reviewed the available literature regarding the psychological aspects of esports using the Preferred Reporting Items for Systematic Review and Meta-Analysis Protocols (PRISMA-P) evidence-based reporting checklist and a Population, Intervention, Comparator, and Outcomes (PICO) framework with the following inclusion criteria: (i) published between 1994 and 2018; (ii) empirical investigation (as the current state of research is dense with positions and opinions but has few empirical investigations); and (iii) focussed on esports games that are associated with either cognitive performance or game performance. The goal of our research was twofold: to present a summary of the empirical evidence addressing the psychological characteristics of both cognitive and game performance in esports, and to integrate esports in the field of sport psychology. More specifically, our goals were to highlight the interplay of psychological aspects of performance and esports and to clearly define the theoretical foundations of the psychological aspects of esports performance. Underlining the differences from video gaming will inform future research directions and stimulate the development of high-quality practice in the applied field of sports and exercise psychology.

41 


\section{Introduction}

Historically, competitive video gaming tournaments started in 1972 at Stanford

University with the game Spacewar (Li, 2016), but the pioneer of professional tournaments is the Cyberathlete Professional League, which started in 1997 (Goodale, 2003). These tournaments represent the first step towards establishing a professional electronic sports (esports) culture. The first recognition by a professional sports governing body took place in South Korea in 2000 when the Korea e-Sports Association was founded as part of the Ministry of Culture, Sports, and Tourism (Korea e-Sports Association, 2014). By the 2010s, esports had experienced a rapid rise, as teams and professional tournaments were founded and broadcasts became common (Popper, 2013; Tassi, 2012).

Even though the professional growth of esports as a performance environment continues, this is in clear contrast to the development of scientific knowledge on the factors involved in high-level esports performance (Campbell, Toth, Moran, Kowal, \& Exton, 2018). For instance, there is increasing scientific research on video games (Anderson et al., 2010; Reeves, Brown, \& Laurier, 2009; Witkowski, 2012; Yee, 2006), but not specifically on esports, where so far, the research is rather qualitative and exploratory (Hallmann \& Giel, 2018). Hence, there is a need for experimental research on esports (Murphy, 2009), starting with a clear differentiation of the prerequisites of video game and esports performance (Dale \& Green, 2017).

Overall, the role of psychological factors in esports is still poorly understood. To address this, we systematically reviewed the esports literature with two main aims: first, to systematically summarize the available literature on esports related to both cognitive and game performance, and second, to integrate esports in the field of sports psychology, more specifically, highlighting the role of psychological aspects of 
performance in esports. Our analysis is based on the building blocks of performance conceptualized in sports psychology (e.g., emotion, motivation, perception, action, and cognition; Raab, Lobinger, Hoffmann, Pizzera, \& Laborde, 2015).

\section{Transition From Video Games to Esports}

Previous research on video games categorised many video games as action video games (Dale \& Green, 2017; see Table 1) based on key characteristics of the game, for example, aiming, shooting, and running (Bediou et al., 2018), despite the games being in accepted genres of their own, for instance, first-person shooters (FPSs; e.g., Counter-

Strike $^{1}$ ), multiplayer online battle arenas (MOBAs; e.g., League of Legends; LoL), real-

time strategy games (e.g., StarCraft), sports games (e.g., FIFA), or fighting games (e.g.,

Marvel vs. Capcom). Other well-known video game genres include role-playing games

(RPGs), simulations, and puzzle games (Lemmens \& Hendriks, 2016). Most of the genres and the video games within them share similar characteristics (e.g., within MOBA: LoL and Dota).

However, the transition of video games from recreational and unstructured use has evolved into a highly competitive domain that is now called professional esports.

This evolution is changing not only how the video games' community interacts but also

87 the way how empirical evidence is obtained in this field (Tang, 2018). For examples, researchers who have studied the effects of video games on cognition acknowledged that the effects found so far could be uncertain if the individual characteristics and the constant evolution of each video game and gamers is not considered (Dale \& Green, 91 2017; Momi et al., 2018). Consequently, one can see how over the years, the development of professional esports has positioned teams and players within a performance context, facilitating the

\footnotetext{
${ }^{1}$ Full names of all games discussed in this review can be found in Table 1.
} 
94 initial understanding of psychological factors underlying esports expertise (Campbell et al., 2018; Himmelstein, Liu, \& Shapiro, 2017; Pedraza-Ramirez, 2019).

\section{Resolving the definitional dilemma in esports}

It is important to mention that there is still a diverse number of spellings and definitions for esports. Therefore, we decided to address and contribute in this matter, aiming to concur in the terminology for future works.

First, we have decided to use the Associated Press's spelling of the competitive video gaming industry (Esports or eSports?, 2017): esports. Second, there are several

103 definitions of the term, which still lack specificity. For example, esports has been

104 defined as the form of competitive videogame playing against other players in person or online, playing for trophies or points, and playing for speed (i.e., competing for the fastest completion time in a game; Ruvalcaba, Shulze, Kim, Berzenski, \& Otten, 2018, p. 296). Additionally, Hamari and Sjöblom (2017, p. 213) defined esports as 'a form of sports where the primary aspects of the sport are facilitated by electronic systems; the

109 input of players and teams as well as the output of the eSports system are mediated by

110 human-computer interfaces'. Even though this definition highlights the term 'sports', it

111 can be debated whether esports require the same physical exertion as mainstream sports,

112 such as football, tennis, or basketball. Additionally, according to the Council of Europe

113 (2001), the word 'sports' refers to 'all forms of physical activity which, through casual

114 or organised participation, aim at expressing or improving physical fitness and mental

115 well-being, forming social relationships or obtaining results in competition at all levels'.

116 However, we would argue that the physical exertion (i.e., energy expenditure, physical

117 effort) in esports during competition and as part of participants' training can indeed be

118 considered similar to that in other activities such as archery, shooting, bridge, or chess 
119 (Schwarz, Schächinger, Adler, \& Goetz, 2003; Troubat, Fargeas-Gluck, Tulppo, \&

120 Dugué, 2009), which are all recognized as sports by the International Olympic

121 Committee.

122 Taking into account the different existing perspectives and the characteristics of

123 esports, we propose the following definition that clearly differentiates esports from

124 video gaming:

125 Esports is the casual or organized competitive activity of playing specific video

126 games that provide professional and/or personal development to the player. This

127 practice is facilitated by electronic systems, either computers, consoles, tablets,

128 or mobile phones, on which teams and individual players practice and compete

129 online and/or in local-area-network tournaments at the professional or amateur

$130 \quad$ level. The games are established by ranking systems and competitions and are

131 regulated by official leagues. This structure provides players a sense of being

132 part of a community and facilitates mastering expertise in fine-motor

133 coordination and perceptual-cognitive skills, particularly but not exclusively, at

$134 \quad$ higher levels of performance.

135 So, according to our definition, not every video game is an esports game but every

136 esports game is a video game. Video games such as Super Mario Bros., The Sims, or

137 Grand Theft Auto can be played casually, and in some cases, there are organized

138 tournaments. But these types of games do not have ranking systems, and competitions

139 regulated by official leagues.

140 The Psychology of Esports Performance

141 In sports psychology, researchers work to understand the underlying

142 mechanisms of performance in different competitive domains (Ericsson, Krampe, \&

143 Tesch-Römer, 1993) within the sports context (e.g., Côté, Baker, \& Abernethy, 2007; 
144 Starkes \& Ericsson, 2003). Accordingly, Murphy (2009) suggested that the physical

145 (motor) and cognitive skills development that occurs when engaging in competitive

146 video games could be of interest to sports psychologists. Consequently, we suggest that

147 esports is an ideal domain for studying performance, and that this domain should be

148 integrated in the research and applied field of sports psychology. In this review, we use

149 cognitive and in-game performance measures to conceptually define and quantify

150 esports performance.

151 High-level performance has received much attention in applied and research

152 sports psychology, with research groups, journals (Sport, Exercise, and Performance

153 Psychology), and books (e.g., Performance Psychology by Raab et al., 2015) devoted to

154 the topic. Performance can be seen everywhere, and it is often used as an umbrella term

155 to explain behaviour associated with the achievement of goals (Raab et al., 2015). Thus,

156 esports performance, similar to physical sports performance, is an end outcome

157 achieved across time that can be measured in different ways (e.g., winning or acquiring

158 gold, being ranked, etc.). From a philosophical perspective, Nitsch and Hackfort (2015)

159 addressed the importance of understanding the psychology of performance from two

160 different angles: First, performance can be seen as the realization of a performance

161 action driven by the motivation and interests of the person, that is, "performance as a

162 means to an end' (p. 13); second, performance can be seen as the realization and

163 perfection of that performance action in itself, that is, 'performance as an end in itself'

164 (p. 13). A competitive esports player requires the cognitive processes needed to meet

165 the demands of the game (e.g., decision making, attention, and memory; Raab et al.,

166 2015; Voss, Kramer, Basak, Prakash, \& Roberts, 2010) and in-game skills (e.g., fine-

167 motor coordination, game knowledge, etc.), which Donaldson (2017, p. 427) called

168 'mechanical expertise'. 
171 theoretical perspectives. Some have focussed on experts' performance, in particular on

172 sport-specific cognitive processes (Musculus, Ruggeri, Raab, \& Lobinger, 2019; Raab,

173 Masters, \& Maxwell, 2005; Voss et al., 2010), and others have taken a more general

174 approach, focussing on cognitive processes that are common to all sports (Voss et al.,

175 2010). In esports, the majority of studies have so far focussed instead on esports-general

176 cognitive processes (e.g., Seya \& Shinoda, 2016). Given this lack of specificity in

177 studies of the cognitive processes of certain video games (Bediou et al., 2018; Green \&

178 Bavelier, 2015), the cognitive processes underlying performance in esports are still

179 unclear. The characteristics of the environment in which esports take place may offer

180 improved ecological validity over laboratory-based research on traditional sports when

181 exploring specific cognitive processes (Pluss et al., 2019). Yet, it is of utmost

182 importance to determine if there is already empirical work testing cognitive processes in

183 laboratory settings that could shed light on esports cognitive performance.

184 Game Performance in Esports

185 As in any other sports domain, the evaluation of game performance is

186 fundamental to understanding progress and the attainability of desired outcomes.

187 Usually in sports, such assessments are based on statistical and outcome parameters

188 (Tenenbaum \& Filho, 2016). In esports, it is possible to measure performance on the

189 basis of results (i.e., win/lose) and in-game statistics (e.g., amount of gold acquired per

190 minute, number of kills, deaths, assists [known in the games as KDA], points, etc.;

191 Bertran \& Chamarro, 2016) or other indicators such as positioning in league rankings or

192 points. However, these game performance measures vary depending on the game.

193 Multiplayer Online Battle Arena games have a very similar structure, and thus similar 
194 outcome measures (e.g., gold per minute, KDA, etc.) can be used. Similarly, FPS games

195 use some of the MOBA game performance measures, such as kills, and deaths (KD)

196 (Parshakov, Coates, \& Zavertiaeva, 2018). However, it is important to acknowledge that

197 even though esports can provide many different statistical measures coming directly

198 from the games, these measures are still too unexplored and unreliable to lead to an

199 understanding of the underlying cognitive processes on their own, so other measures of

200 performance are still needed. Accordingly, we would like to consider these in-game

201 performance outcomes as esports performance.

202 Previous Reviews and Meta-Analyses of Esports

203 Esports is a growing industry, and as yet, few theoretical models of the

204 fundamental mechanisms of competitive performance have been developed. Although

205 there are still not enough empirical studies to compare effect sizes in the field of

206 esports, there are a few published meta-analyses of video game research and cognitive

207 abilities. For example, Bediou and colleagues (2018) focussed on the impact of action

208 video games on cognition. Medium effect sizes were found in cross-sectional studies of

209 habitual action video game play, whereas in intervention studies a small to medium

210 effect size was found in a few cognitive domains. Overall, Bediou and colleagues

211 (2018) suggest that those who regularly play action video games display better

212 cognitive processes than those who play little or none. Additionally, authors, concluded

213 that the positive enhancements in cognition do not equally impact all cognitive

214 functions. Thus, the cognitive processes associated with working memory and

215 inhibitory control within perception, spatial cognition and top-down attention seem to

216 show considerable promises in both the theoretical and practical understanding of action

217 video games and cognition.

218 In another meta-analysis, Sala, Tatlidil, and Gobet (2017) concluded that video 
219 game training does not enhance cognitive abilities. The overall results showed, first,

220 weak correlations between skill and cognitive abilities; second, small differences in the

221 cognitive abilities between players and non-players; and third, no or insignificant effects

222 between a video game training group and a control group. These findings do not support

223 the theories of far transfer of video game training and cognitive training. However, there

224 is the need of advanced experimental designs that allow us to better understand any far

225 transfer effects or specific cognitive processes that are important for specific video

226 games. In contrast, Wang and colleagues (2016) aimed to assess the effects of action

227 video game training on cognitive abilities. They found moderate to small effect sizes in

228 overall and specific cognitive improvement in healthy adults. Especially, improvements

229 were shown in cognitive processes related to inhibitory control, such as visuospatial and

230 attention processing. Similarly, a study testing the impact of video games on

231 information processing (Powers, Brooks, Aldrich, Palladino, \& Alfieri, 2013) found that

232 in true experiments, video game training had a significant (small) effect on inhibition,

233 whereas quasi-experimental studies showed moderate effects for dual/multitasking and

234 switching, and small effects for inhibition, intelligence, and working/short-term

235 memory. Even though the results from the quasi-experimental studies showed higher

236 impact of action video game training on the four cognitive functions (e.g., working

237 memory, inhibitory control, cognitive flexibility, and higher order), it is suggested that

238 specific cognitive processes are closely related to the cognitive demands of the game

239 used.

240 The above discussed results shed light on the importance to consider separately

241 the cognitive demands of each video game to better understand the specific cognitive

242 processes that could be more or less impacted by video game training. 
Although the findings of these meta-analyses contribute to the understanding of the cognitive effects of video games in general, methodological limitations (publication bias, small effect sizes, and conceptual issues across different meta-analytic comparisons) have been acknowledged (Bediou et al., 2018). When considering esports, even if it is too soon to discuss the effects of training, the evidence from the metaanalyses in video games seems promising to understand key cognitive processes such as perception, spatial cognition, attention, intelligence and dual/multitasking in specific games that require high cognitive load (e.g., LoL, CS:GO, StarCraft, etc.).

Moreover, there are three important literature reviews that have highlighted the need to develop scientific knowledge on the psychological and cognitive concepts of esports.

Bányai, Griffiths, Király, and Demetrovics (2019) explored the relationship between esports and psychology. Eight studies were included and categorised into three main topics: (1) becoming an esport player, (2) the characteristics of esport players, and (3) the motivations of esport spectators. The authors concluded that the path of becoming an esport player is similar to that of a professional athlete in physical sports

259 (e.g., training, preparation, mental skills, and obstacles). Furthermore, the authors argued that professional video gamers and professional gamblers have similarities, such as excessive time spent playing. Consequently, the authors suggested that future research should consider these similarities and focus on esport players' psychological vulnerability. Garcia-Naveira, Toribio, Molero, and Suarez (2018) reviewed 26 articles on the cognitive and psychological benefits of video games and esports. They concluded that

266 the regular practice of video games and esports stimulates specific brain structures and 267 benefits the development of cognitive processes (e.g., intelligence, working memory, 
decision making, cognitive flexibility, etc.). Also, this practice was found to positively influence psychological skills such as motivation, self-regulation, self-confidence, and social skills. The authors acknowledged the need to study video games and esports separately, yet they included both in their review.

Lastly, Mora-Cantallops and Sicilia (2018) explored player behaviour in MOBA games, mainly focussing on two esports, LoL and Dota 2. This review restricted the search to articles on MOBA games published since 2011. The authors found 23 studies attempting to understand the behaviour and motivation of players, describe social interactions in the online world, and gain knowledge of game play and outcomes using computer modelling, topological measures, and spatio-temporal behaviours of the teams. The review concluded that researchers should cooperate with professional players to better understand tactical and strategy of the games to be able to combine traditional research approaches like survey and interviews with innovative computer science techniques.

Although Bányai et al.'s (2019) summary of the qualitative research on esports players is appreciated, our contribution is aimed at looking into only the quantitative evidence in relation to esports and performance. Additionally, whereas Garcia-Naveira et al. (2018) looked only at the research on the benefits of video games in general since 2012, we undertook a more specific exploration of both the cognitive and in-game outcomes of playing, and, as suggested by Garcia-Naveira et al. (2018), we included not

288 only esports that were developed shortly before or after the 'boom' in esports in 2010

289 (e.g., LoL; Dota 2) but also those that have been around much longer, such as Tetris since 1984. Thus, we were not limited by the databases' (see Information sources,

291 below) maximum range (1994-2018) but could also include studies identified through 292 other sources published before or after that range, in contrast to both Garcia-Naveira et 
al. (2018) and Mora-Cantallops and Sicilia (2018). Although they followed specific

294 social science guidelines for systematic reviews (Petticrew \& Roberts, 2008), Mora-

295 Cantallops and Sicilia (2018) explored a wide range of topics not necessarily related to

296 esports performance. We have focussed on integrating esports performance in sports

297 psychology and have also followed the Preferred Reporting Items for Systematic

298 Review and Meta-Analysis Protocols (PRISMA-P) guidelines and the Population,

299 Intervention, Comparator, and Outcomes (PICO) study design model for reporting

300 systematic reviews and meta-analyses (Liberati et al., 2009; Shamseer et al., 2015).

301 The Current Systematic Review

302 Our systematic review yielded the added value of clarifying and highlighting

303 relevant factors of cognitive and in-game performance in specific esports that have been

304 previously studied in general video game research without considering the relationship

305 of competition and performance in esports. Additionally, we provide a heuristic model

306 to illustrate the current state of the art, suggesting that future research should test the

307 bidirectional influence of all factors related to esports performance.

308 Methods

309 Eligibility Criteria

310 Studies were selected according to the PICO criteria (Table 2). The literature search

311 covered the period of January 1994 (the earliest date allowed in one of the databases) to

312 October 2018 (the point at which we conducted our search). Since one of the databases

313 limited the period of our search (i.e., no earlier than 1994) and we wanted to include all

314 relevant publications, we were slightly flexible and included records identified through

315 other sources (see Information sources) that were published before or after that range.

316 To ensure quality, our selection criteria were publications in a peer-reviewed journal

317 and being written in either English or Spanish. 


\section{Information Sources}

The literature search was conducted in the electronic databases Web of Science,

321 Science Direct, and EBSCOhost. The latter allowed us to narrow the search to the most

322 relevant databases for our interest (SPORTDiscus, PSYNDEX, and SocINDEX).

323 Additionally, the reference lists from all selected papers and the respective citations of

324 each study from the time of publication up to the date of our search were hand searched.

325 Even though we did not include unpublished studies (e.g., conference posters and

326 abstracts, theses, etc.), books, or position papers in or analysis, the reference lists from

327 those sources as well as reviews and meta-analyses were checked and considered to

328 establish the state of the art.

\section{Search Strategy}

330 We searched for only quantitative studies, using terms combined with different

331 expressions (esports OR e-sports OR electronic gaming OR competitive gaming OR

332 online gaming OR professional video gaming) AND (psychology OR cognition OR

333 psychological skills OR performance OR neural basis). The combination of such terms

334 was applied in each of the databases (i.e., Web of Science, Science Direct, and

335 EBSCOhost).

\section{Study Records}

\section{Data Management}

338 The first phase of the literature search involved exporting the results

339 (title/abstract) to a Word document to determine which studies were potentially relevant

340 to our systematic review. The first author screened the titles and abstracts to remove any

341 that did not meet our inclusion criteria, after which the selected studies were uploaded

342 to the reference manager software Mendeley to check for duplicates. The results were 
exported to an Excel spreadsheet, where the articles were numbered. Subsequently, the

titles and abstracts went through a second phase consisting of extracting initial

345 information from each article in the Excel spreadsheet (e.g., study title, author, year,

346 journal, the aim of the study, etc.) while verifying once more whether the article met the

347 selection criteria.

\section{Data Collection Processes}

349 Using the Excel spreadsheet, the first author extracted data that included

350 demographic information, design information, performance measures, the aim of the

351 study, the intervention details, and the outcomes. Each study that was included in the

352 final pool and any whose inclusion was unclear after the selection criteria assessment

353 were discussed in a team meeting, by at least two more authors, until consensus was

354 reached. Figure 1 shows a flow diagram of the selection process, following the PRISMA-P methodology. Fifty-two studies were included for final analysis.

[Figure 1 near here]

\section{Data Items}

Some studies considered for inclusion focussed on specific esports but others on many different video games, so we considered only those studies that clearly addressed esports. Also, some focussed on either one or both performance outcomes (i.e., game performance and cognitive performance). Therefore, we extracted the specific measures used. Lastly, we conducted a search on the journal names to get a better understanding

363 of where the initial research on esports performance has been published.

\section{Outcomes and Prioritization}

The main outcomes of our review were (1) the types of esports video games analysed in the studies, which were dependent on our esports definition, and (2) esports

367 performance outcomes: (a) the performance on cognitive tasks (cognitive processes 
tested in a laboratory setting or controlled environment) and (b) the game performance measures coming from the games themselves (e.g., winning/losing, KDA, gold per minute, etc.).

\section{1}

\section{Risk of Bias in Individual Studies}

Risk of bias within the included studies was assessed by two of the authors using the Joanna Briggs Institute Critical Appraisal Checklist for Analytical Cross Sectional studies (Moola et al., 2017). This tool contains eight risk-of-bias questions to determine the extent to which the selected studies addressed the possibility of bias in their design, running, and analysis. Using validated definitions, each item was answered with yes, no, unclear, or not applicable (see Appendix A).

\section{Results}

\section{Study Description}

The $N=52$ publications that met the inclusion criteria (see Figure 1) were published between 1992 and 2019. The studies were published in many different journals $(N=40)$. PLOS ONE, with six, published the most, and four were published in Computers in Human Behavior. For the cognitive science journals, Applied Cognitive Psychology published four and Topics in Cognitive Science three. The majority of studies $(n=35)$ were the only publications on the topic in the respective journal (e.g., Nature, Proceedings of the National Academy of Science of the United States of America, etc.; see Appendix B). The studies were divided into the two main categories of esports performance: (a) cognitive performance and (b) game performance. An extra category was created for studies combining the two measures. The main findings of the systematic review are presented in Table 3 for cognitive performance and Table 4 for game performance. Overall, $25 \%(n=13)$ of the research on esports performance targeted only cognitive performance, $46.2 \%(n=24)$ targeted only game performance, 
and $28.8 \%(n=15)$ reported both of these characteristics. publications were divided by the different cognitive functions they assessed (Diamond,

399 2013; Miyake et al., 2000): (a) working memory $(n=8)$, (b) inhibitory control $(n=2)$,

(c) cognitive flexibility $(n=2)$, (d) higher order functions, and any combination of two or more $(n=16$; Table 3$)$. The publications on game performance $(n=39)$ were of an experimental $(n=14)$, quasi-experimental $(n=6)$, cross-sectional $(n=13)$, longitudinal $(n=3)$, experimental and cross-sectional $(n=1)$, quasi-experimental and crosssectional $(n=1)$, or cross-sectional and longitudinal $(n=1)$ design. These publications were divided into two main categories that enabled us to differentiate the purpose of the studies (following Nitsch \& Hackfort, 2015): (a) expertise differences (i.e., esports performance as a means to an end; $n=20$ ) and (b) effects of esports participation/training (i.e., esports performance as an end in itself; $n=19$; Table 4).

\section{Cognitive Performance}

The studies measuring cognitive performance used a great variety of cognitive

411 tasks to understand the link between esports and cognitive functions (e.g., Boot,

412 Kramer, Simons, Fabiani, \& Gratton, 2008; Kokkinakis, Cowling, Drachen, \& Wade,

413 2017; Table 3). For example, the most frequent cognitive tasks used by authors to assess

414 working memory were mental rotation tasks $(n=6)$ and the operation span task $(n=6)$;

415 inhibitory control was investigated with the useful field of view $(n=4)$ and multiple

416 object tracking $(n=4)$ tasks, cognitive flexibility with task-switching paradigms $(n=3)$, 417 and higher order functions with Raven's matrices $(n=3)$ and the Wechsler Abbreviated 
418 Scale of Intelligence $(n=2)$.

419 Working Memory

420 Overall, mixed effects were reported regarding the relationship between esports

421 training and working memory. That is, positive effects of training/participation were

422 found in working memory tasks such as spatial resolution and mental rotation in Unreal

423 Tournament, Tetris, CoD, and CS:GO (C.S. Green \& Bavelier, 2007; Lau-Zhu, Holmes,

424 Butterfield, \& Holmes, 2017; Momi et al., 2018; Okagaki \& Frensch, 1994; Seya \&

425 Shinoda, 2016; Terlecki, Newcombe, \& Little, 2008), but some counter-evidence exists

426 of the effects of esports training in working memory tasks in Dota 2 and Tetris (Boot et

427 al., 2008; Pilegard \& Mayer, 2018; Röhlcke, Bäcklund, Sörman, \& Jonsson, 2018).

428 In terms of expertise differences, researchers found that expert LoL, Dota,

429 StarCraft, Guilty Gear, CoD, Halo, and Battlefield players outperformed amateur

430 players in spatial and visual working memory tasks (Chang, Liu, Chen, \& Hsieh, 2017;

431 Gong et al., 2016; C.S. Green \& Bavelier, 2006; Kowalczyk et al., 2018; Pereira,

432 Wilwert, \& Takase, 2016; Seya \& Shinoda, 2016; Tanaka et al., 2013). Additionally,

433 studies found higher activation of the brain network structure associated with spatial

434 and visual processing of the working memory functions in expert Guilty Gear, LoL,

435 Dota, and StarCraft players in comparison to non-expert players (Gong et al., 2016;

436 Kowalczyk et al., 2018; Tanaka et al., 2013). In sum, although there are exceptions,

437 positive effects of training and expertise differences on working memory have been

438 observed in a great variety of esports.

439 Inhibitory Control

440 In terms of inhibitory control, positive effects of esports training were found in

441 LoL, Unreal Tournament, CoD, Halo, Counter-Strike, Gears of War, and Tetris players

442 in comparison to non-players on visual selective attention and multiple-object tracking 
tasks (Bavelier, Achtman, Mani, \& Föcker, 2012; Bejjanki et al., 2014; Ding et al.,

444 2018; C.S. Green \& Bavelier, 2006; Qiu et al., 2018). Additionally, brain networks

445 associated with inhibitory control, in expert LoL, Dota 2, and FIFA 15 players showed

446 enhanced functional integration between salience and central executive networks of the

447 brain and stronger activation in comparison to non-experts (Aliyari et al., 2015; Ding et

448 al., 2018; Gong et al., 2016). Furthermore, Halo, Counter-Strike, Gears of War, and

449 CoD players had faster reaction times and presented lower activity in the frontoparietal

450 network that mediates attention location in comparison to non-players (Bavelier et al.,

451 2012). Overall, positive effects of esports training on inhibitory control processes were

452 found in different types of esports, especially in those that require fast reaction times,

453 such as Counter-Strike and $\mathrm{CoD}$, but also those in which strategy and planning are

454 highly important, such as LoL and Dota 2.

455 Cognitive Flexibility

456 Overall, only a few studies have explored the relationship between esports

457 training or expertise and cognitive flexibility (e.g., Glass, Maddox, \& Love, 2013; Hyun

458 et al., 2013; Klaffehn, Schwarz, Kunde, \& Pfister, 2018). The main findings were found

459 in StarCraft I and II for long-term engagement and training. Researchers found an

460 increased volume of the prefrontal cortex and positive significant correlations of

461 training and performance on cognitive flexibility tasks (Glass et al., 2013; Hyun et al.,

462 2013). However, in a study with many different types of esports, Klaffehn et al. (2018)

463 found that Age of Empires, LoL, Dota, StarCraft II, Battlefield, CS:GO, CoD, and

464 Counter-Strike players did not show higher task-switching performance in comparison

465 to non-players. 


\section{Higher Order Functions}

Mixed evidence has been reported regarding the relation between esports and

higher order functions. Esports training and practice in expert and non-expert Unreal

Tournament, CoD, Tetris, and LoL players were found to be associated with an improvement in fluid intelligence and higher order function tasks (Bejjanki et al., 2014;

Kokkinakis et al., 2017; Lau-Zhu et al., 2017): Conversely, other studies of Dota 2 and

472 Tetris players found experience had no effect on fluid intelligence (Boot et al., 2008;

473 Röhlcke et al., 2018).

\section{Game Performance}

Thirty-nine studies investigated expertise variables of in-game performance or the effects of esports participation or training by using specific in-game variables (e.g., results, KDA, and ranking) to observe game-playing effects or to measure engagement

478 (e.g., Boot et al., 2008; Breuer, Scharkow, \& Quandt, 2013; C.S. Green \& Bavelier, 2007; Huang, Yan, Cheung, Nagappan, \& Zimmermann, 2017; Table 4).

Expertise Differences in Esports Performance

Several studies reported that expert LoL and Tetris players were faster and more accurate in decision-making situations and better than non-experts at performing under high pressure, as measured via biosignals of the autonomic nervous system (e.g., heart rate variability, and respiration rate; Ding et al., 2018; Lindstedt \& Gray, 2019; Maglio,

Wenger, \& Copeland, 2008). Furthermore, during a simulated tournament, higher cortisol levels were found for high-ranked Unreal Tournament players immediately

487 before and after a winning match (Oxford, Ponzi, \& Geary, 2010). Conversely, measures of game experience and performance in LoL players were unrelated to hormone changes (e.g., cortisol, testosterone, etc.) between playing against humans 
compared to playing against machine (i.e., artificial intelligence) (Gray, Vuong, Zava, \& McHale, 2018). significantly better than non-experts at in-game tasks such as mini-map recall, players allocate more time to look at certain regions of the map such as HP/Mana and Shop Button, extrapolating information with one fixation, distinctive use of Hotkey Selects (i.e., keys combination; shortcuts) and the levels of Action Latency (i.e., interval between action and response) (Bonny \& Castaneda, 2016; Castaneda, Sidhu, Azose, \& Swanson, 2016; Thompson, Blair, Chen, \& Henrey, 2013). In Tetris, novice players' ability to place the Zoids (i.e., blocks) seemed to show a lack of the perceptual-motor

500 skills involved in planning and decision making, a difference from experts who are more engaged in the game strategy process (Sibert, Gray, \& Lindstedt, 2017).

503 of practice on expertise. In terms of the amount of practice, authors have found on the

504 one hand that the number of games played in Dota 2 is a strong predictor of expertise 505 (Röhlcke et al., 2018). On the other hand, in LoL, researchers have reported a weak 506 relationship of the number of games played and expertise (Kokkinakis et al., 2017). In 507 regard to the relationship of age and expertise, authors have suggested that there is a cognitive-motor decline in StarCraft II players associated with age that seems to begin around 24 years (Thompson, Blair, \& Henrey, 2014). Also, it has been suggested that in

510 Destiny, Battlefield, LoL, and Dota 2, peak performance is experienced at around 22-27

511 years old (Kokkinakis et al., 2017). However, findings have shown that performance in

512 Battlefield 3 peaks around the age of 20 and that older players show weaker

513 performance and offset this deficiency by practicing more (Tekofsky, Spronck,

514 Goudbeek, Plaat, \& van den Herik, 2015; Thompson et al., 2014). These mixed results 
515 show that the expertise differences attributable to age and amount of practice should be

516 considered and researched individually for better clarification, as they may be unique to 517 each esport.

518 In terms of practice and learning styles, establishing consistent habits and

519 forming routines were found to be important characteristics of expert Halo, StarCraft II, 520 and LoL players. Research showed that the most effective strategy to improve players' 521 skills included both playing a moderate number of matches and taking short breaks 522 (Huang et al., 2017; Sapienza, Zeng, Bessi, Lerman, \& Ferrara, 2018). Furthermore, 523 Destiny and LoL players who more regularly chose to play with self-selected teammates 524 tended to have higher game performance than those who decided to play with random 525 teammates (Kahn \& Williams, 2016; Pirker, Rattinger, Drachen, \& Sifa, 2018).

526 Sapienza et al. (2018) also found that individual and team performance in LoL can be affected by the presence of friends on a team.

At the team level, authors have identified specific characteristics of successful teams. For example, Xia, Wang, and Zhou (2017) found in Dota 2 that the frequencies 530 of kills by one player, kills by multiple players, initiation of fights, and activation of 531 runes were significantly higher on the winning teams than on the losing teams.

532 Additionally, it was found that the successful teams in LoL were those that showed the 533 highest scores on the Transactive Memory System Scale, which measures a group's 534 ability to learn, remember, and communicate knowledge relevant to the group (i.e., 535 shared knowledge; Kahn \& Williams, 2016). Moreover, Wang, Yang, and Sun (2015)

536 found that those teams that possessed at least one player with a global-liberal playing 537 style, that is, a player who assisted teammates (i.e., collaborative style), had

538 significantly higher win rates than teams without this type of player. 


\section{Effects of Esports Participation/Training}

Several psychosocial characteristics of participation and training in esports have been shown to affect performance. For example, looking into the cooperative and competitive aspects of FIFA, Dota 2, and LoL players' style, researchers found that players' behaviour, attitudes towards winning and losing, and harmonic and obsessive passion had significant effects on affect and performance (Bertran \& Chamarro, 2016; Breuer et al., 2013; Hudson \& Cairns, 2016). In regards to the effects of motives for participating in Dota 2, improvement in solo matches (i.e., no predetermined teams) suggests that skill improvement or the sense of personal achievement were primary motivators for playing (Bonny \& Castaneda, 2017). Furthermore, exploring CS:GO and WoW participation, studies showed gender and game performance interactions as a predictor of enjoyment, with women deriving enjoyment from discovering the games, while men were more interested in the competition aspect of the games (Billieux et al., 2013; Hopp \& Fisher, 2017). Another esports characteristic, the presence of an audience, was shown to decrease players' performance while playing Tetris (Kimble \& Rezabek, 1992). However, a positive association between audience presence and improved performance was discovered for the game Quake (Bowman, Weber, Tamborini, \& Sherry, 2013). Finally, in Doom, Tafalla (2007) found positive effects of playing with the in-game sound on performance.

\section{Discussion}

Beginning in the early 1990s and for almost 30 years since (see Appendix C), researchers have been exploring the psychosocial aspects of competition and cognitive processes in esports. The early works acknowledged that certain video games could facilitate a deeper understanding of human performance. However, it took almost 20 years, from the first study in 1992 until 2010, for 10 studies related to the performance 
564 of what we now call esports to be published. Finally, from 2011 to the beginning of 5652019 , an additional 42 studies were published on esports performance. This systematic

566 review of 52 articles, following the PRISMA-P guidelines, presents the available

567 evidence regarding the psychological aspects of esports performance.

\section{Cognitive Performance in Esports}

569 The mixed effects found for esports training on working memory tasks might

570 have been due to differences in the methodological designs and aims of the studies, with

571 low sample size and the inclusion of many different cognitive tasks possibly affecting

572 the results. The effects of expertise on working memory tasks are in line with the

573 positive effects of esports training and the demands that strategy and FPS esports make

574 in terms of holding and monitoring information. Furthermore, the higher levels of 575 expertise in esports (e.g., LoL, Dota 2, Counter-Strike, etc.) and the activation of the brain networks associated with inhibitory control processes show the importance of information processing for game performance. Although we encourage to understand

578 the specific individual cognitive demands for each esports, it is necessary to

579 acknowledge that there will be an overlap when referring to cognitive functions. For 580 instance, working memory and inhibitory control generally need one another, especially

581 in complex esports such as LoL, StarCraft, and Counter-Strike where there is a vast

582 amount of information available from opponents, teammates, and the game

583 environment. Consequently, players need to assess what information is relevant for their

584 goal, hold it using their working memory capacity, and determine what to inhibit to

585 make favourable decisions.

Despite the mixed evidence found between cognitive flexibility processes and

587 esports, the research is promising to understanding the cognitive demands of changing 588 perspectives and adapting to new information in specific esports. The constant changes 
in esports like LoL (e.g., creation of new avatars, skills, rules, etc.) force players to find ways to adapt and keep up with those changes, which means structure training methodologies and learning styles are important during this process.

593 (Kokkinakis et al., 2017), but not in players of the similar game, Dota (Röhlcke et al., 594 2018). Consequently, these results indicate, first, the importance of considering the uniqueness of each esports (e.g., frequency of game updates) and how this could be associated with specific cognitive demands on players. Second, one must consider the methodological designs of these two studies, which might explain the contrasting results: Röhlcke et al.'s (2018) study that found no effects was not carried out in a

599 controlled environment, which could have affected the reliability of the results.

600 Furthermore, improvements in performance found after Unreal Tournament and CoD

601 training (Bejjanki et al., 2014) might suggest that higher-order functions are related to

602 the more unpredictable and cognitively demanding type of esports. different cognitive processes and motor abilities. Consequently, esports research and sports psychologists will benefit from understanding the underlying cognitive mechanisms of each esports, in order to adapt training strategies to the specificities of each game. For example, some esports are very stable in the way they need to be played across years or months, while in other esports, very specific characteristics can change every 2 weeks (e.g., LoL) and players are required to adjust, adapt, and learn the new

610 demands of the game, which may require higher levels of cognitive flexibility and

611 higher order functions (e.g., fluid intelligence, decision making, etc.) to achieve peak

612 performance. Brain imaging and psychophysiological tools will facilitate this

613 understanding of cognitive processes and possible predictors of performance among 
614 players due to the ecological validity that esports can provide.

615 Even though we agree that there is increasing empirical evidence of the

616 relationship between playing esports and improved cognitive performance, the mixed

617 evidence and methodological limitations cannot be ignored (e.g., Boot et al., 2008;

618 Klaffehn et al., 2018). Consequently, it is necessary to consider the potential

619 confounding variables of the studies included in this review that could affect the

620 reliability of the results, such as the learning curves of participants (Röhlcke et al.,

621 2018; Tekofsky et al., 2015) that varied according to gender and age (Terlecki et al.,

622 2008; Thompson et al., 2014). Thus, we recommend that esports researchers change the

623 methodology of their research to move away from exploring video games as a genre

624 (e.g., action video games, FPS, MOBA, real-time strategy, etc.) and more towards

625 considering individual esports. For instance, although soccer, basketball, American

626 football, and rugby share many similar characteristics, they are individually quite

627 distinct sports. Consequently, while the overall findings on cognitive performance are

628 promising, they reveal that the way forward is to consider the methodological design

629 issues: A more controlled design of the experiments should be considered that takes into

630 account, for example, individual differences, gender, gaming experience, playing

631 frequency, and nature of the game (Boot et al., 2008; Dale \& Green, 2017; Klaffehn et

632 al., 2018). This will lead to a better understanding of task transferability in esports

633 training as well as the cognitive functions underlying performance in each esports.

\section{Game Performance in Esports}

The results of our game performance analysis provide several takeaways related to expertise differences and the effects of taking part in esports. First, the findings

637 highlight specific player characteristics that are important when differentiating 638 expertise, particularly in LoL and Tetris, such as fast and accurate decision making and 
639 performing under pressure (e.g., Ding et al., 2018; Gray et al., 2018). These

640 characteristics are particularly important when players are competing at the highest

641 levels, where different types of pressure (e.g., time, prizes, expectations, audience, etc.)

642 are present and could define outcomes. Second, results show that there are specific

643 psychological and in-game skills that players can develop to achieve higher levels of

644 performance. Skill development, in particular, seems to be an area where sports

645 psychologists could help in the development of expertise in esports players, particularly

646 as relates to deliberate practice (Ericsson et al., 1993). For instance, in LoL, a

647 performance decline occurs when playing sessions are too long (Sapienza et al., 2018),

648 but experienced players showed fewer performance declines over the course of a

649 session than newer players. Supporting this idea, Halo players showed that less intense

650 and frequent training can result in skill development, but breaks that are too long result

651 in a loss of skill (Huang et al., 2017). Coaches and players can benefit from this crucial

652 information to optimise their training programs according to the characteristics of each

653 esports. Since players experience different performance peaks and declines depending

654 on the esports. For example, StarCraft player suffers from performance declines starting 655 at around 24 years of age (Thompson et al., 2014), in LoL and Dota performance peaks 656 are experienced between 22 and 27 years of age (Kokkinakis et al., 2017), and in

657 Battlefield, a shooting game, peaks are seen at an earlier age: 20 years old (Tekofsky et 658 al., 2015). It is important (a) to consider how players are practicing to improve their 659 skills and (b) to understand the requirements and characteristics of each esport, as, for 660 example, in shooting games more reaction time and attention are needed than in strategy 661 games, where extracting information, planning, and decision making may be more 662 important. In terms of skill acquisition, results are promising, as they show it is possible 663 to implement training models that aid the development of players. 
Psychosocial characteristics in esports have been considered to understand how participation or training is related to player behaviour, motivation, affect, and performance. Thus, coaches need to adapt their training methodologies to fulfil players psychological needs, considering the differences in the effects of cooperation and competition concerning gender seen in CS:GO and WoW. Also, in Dota 2 the sense of personal achievement and skill improvement were primary motivators to play (Bonny \& Castaneda, 2017). Additionally, the impact of winning and losing in WoW (Hudson \& Cairns, 2016) and the psychological characteristic of harmonic and obsessive passion in needs of autonomy, competence and relatedness in relation with future game results.

674 This may be an area where sports psychologists can offer interventions tailored to specific players and the competitive characteristics of esports. Thus, acknowledging the impact of social-cognitive and affective behaviour on esports players could facilitate learning and the optimisation of performance (Wulf \& Lewthwaite, 2016). methodological design that acknowledges specific variables that can alter performance, such as considering randomised and blinded experiments, gender and age differences, game history, and the so-called hybrid games (i.e., games with shared characteristics of two or more genres; Dale \& Green, 2017). Similarly, authors have suggested that some games share identical characteristics across genres (e.g., FPS, MOBA, etc.), but some

684 have unique elements that need to be considered individually (e.g., CS:GO; Dale \& 685 Green, 2017; Momi et al., 2018). Also, the biosocial influence of competition has to be considered, as playing against the artificial intelligence of the game (i.e., bots) is not the same as playing against humans. 
It is necessary to better understand expertise and the performance indicators in esports that could support this developmental process. Consequently, having set the scientific stage for esports psychology, first, future investigations should consider recent appeared papers that may be relevant for the development of esports and its alignment

692 with sport psychology (e.g., Gong, Ma, Liu, Yan, \& Yao, 2019; Maciej, Kosakowski, \&

693 Kaczmarek, 2020; Thompson, McColeman, Blair, \& Henrey, 2019). Second,

694 investigations should test the bidirectional influence of the factors related to esports performance and the strength of their influence and should explore those factors that are theoretically plausible but empirically not yet tested. Such as the interplay of high order

697 functions like decision making and affect to understand expertise differences in esports

698 performance. Thus, we provide a heuristic model to illustrate the current state of the art 699 (see Figure 2).

\section{Conclusion}

The present systematic review highlights that research in esports could greatly benefit from a closer alignment to the field of sports psychology, leading to a better understanding of the underlying mechanisms of performance, especially by focussing on cognitive functions and game performance. By integrating the two disciplines, each can help the other overcome theoretical and methodological constraints. For example, esports as a field of research can help efforts to reveal cognitive processes involved in

708 performance and understand individual development and can serve as a domain for the use of neurophysiological markers. This review provides a starting point for future research endeavours, because it is the first systematic review following the PRISMA-P

711 guidelines that explores and separates the empirical evidence on general video games

712 (from their inception) from evidence on esports. Although the research in this field is 
713 promising, much work remains. Therefore, to promote the development of research on esports performance we offer our list of the top 10 challenges esports performance research will likely face in the future.

\section{Top 10 Future Challenges of the Psychology of Esports Performance}

(1) Implementing rigorous methodological designs: The research in esports needs to acknowledge the experimental design constraints, already addressed by video game researchers (Dale \& Green, 2017), and implement appropriate methodological designs, for instance, avoiding grouping games according to genres, using randomised and blinded experimental designs, and implementing the appropriate statistical power. Also, there is a need to consider homogeneous sample issues (i.e., females and males) and to apply a rigorous criterion for age and video game experience, given the influential differences in cognitive and

(2) Acknowledging the impact of cognition in esports performance: As shown in this review, different esports rely on different cognitive functions and at times the cognitive requirements are unclear. Thus, it is important to understand (a) how cognitive demands change across different esports and how specific cognitive functions could be more relevant for specific games, (b) what specific esports measures could be defined to understand performance, and (c) the contribution of the building blocks of psychology, that is, perception, memory, emotion, and cognition, to esports performance (Raab et al., 2015).

(3) Identifying performance indicators: Any esports offers a great range of statistical variables that could be connected to player performance. However, these variables can be misinterpreted, generating incorrect information related to performance. Therefore, it is necessary to understand (a) how game performance 
characteristics change across games, and (b) what reliable data or performance measures will lead to an accurate understanding of esports expertise. For instance, in sports expertise, differences have been revealed by the decision making of athletes (Musculus, 2018). Additionally, in auto racing, Formula 1 drivers are known for using telemetry information to optimise the performance of the car; using telemetry in esports (see Thompson et al., 2017) could be an interesting way to understand the great number of parameters that can potentially provide performance indicators of esports, ultimately improving esports players' and coaches' cognitive and motor processes for competition.

(4) Building expertise in esports: In sports, deliberate practice has been used to help players systematically optimise improvement and achieve higher levels of expertise (Ericsson, 2019). However, esports is still working on finding reliable systems that help players and coaches improve performance (Green, 2018). Therefore, a big challenge will be to provide adequate tools and structures to help players and coaches learn and improve performance. One example could be to apply the principles of motor learning theory (OPTIMAL; Wulf \& Lewthwaite, 2016), in which performance and learning are thought to be influenced by positive motivational and attentional focus. This approach enables players with a safe environment for satisfaction of the psychological need for competence, establishing situations for choice and a sense of autonomy, which

(5) Defining career development of esports players: The current route to excellence in esports is still unclear. So, there are no developmental stages defined or real career plans (Abbott \& Collins, 2004). Thus, one of the roles of 
sports psychology in esports should be to facilitate (a) the identification of players' career paths, and (b) the adaptation and transferability of skills during different transition stages that esports may have (e.g., leagues, countries, different esports, retirement, etc.). This support can facilitate talent development and proper institutionalization of esports as a performance domain.

(6) Addressing the needs of coaches and coaching development: The proper creation of training models that coaches can implement or adapt to their daily practices supports talent development. However, before that, understanding how esports skill acquisition and development work is necessary. Also, educating coaches on how to coach is crucial. Thus, establishing esports coaching courses could be a good first step. Supporting and developing places where coaches could develop themselves, such as in academy teams, could facilitate developmental processes at the professional level where the performance pressure is higher. Providing more opportunities for research and applied sports psychology would help sustain the healthy evolution of esports (Cottrell, McMillen, \& Harris, 2018). Thus, the requirement of certification for coaches

(7) Stopping opportunistic esports enhancement tools: The development of lucrative opportunistic tools will be likely to increase in the applied field. For example, the large number of publications in low-quality journals and the amount of grey literature in esports is rapidly expanding. Also, the development of cognitive training devices to improve performance has earned a bad reputation. While it is very useful to understand the foundations of esports performance and use innovative tools, it is necessary to avoid the support of unreliable non-scientific knowledge. Thus, there is a need for more quality 
research to develop a better understanding of cognitive training in sports (as suggested by Walton, Keegan, Martin, \& Hallock, 2018).

(8) Integrating machine learning models: Motor learning is an important internal process in the development and retention of skills in sports. This is characterised by a set of stages in which cognition is highly relevant to determining appropriate strategies that influence learning processes (e.g., mental practice, observational learning; Schmidt, Lee, Winstein, Wulf, \& Zelaznik, 1999). In esports, the proper understanding and use of algorithms and statistical models for performance could greatly advance the development of psychological and cognitive tools that can positively impact performance and training methodologies and facilitate the comprehension of the cognitive and motor behaviour of esports expertise (Lindstedt \& Gray, 2019). However, suitable comprehension and multidisciplinary collaboration are essential to make good use of technological advances.

(9) Developing knowledge from biological markers: The high ecological validity of the esports setting for neurophysiological research of performance is undeniable. Consequently, developing an understanding of the psychophysiological and neuropsychological aspects of esports players is an appealing avenue of research for the scientist (Campbell et al., 2018; Murphy, 2009). Thus, future research on biological markers should be carried out. Understanding the interplay of cognitive and behavioural performance in esports could be facilitated by studies on, for instance, hormone responses and heart rate variability and by making use of brain stimulation techniques, eye tracking, and brain activation measures such as electroencephalography and functional magnetic resonance imaging. 
813 (10) Understanding the impact of a rapidly changing esports system: Esports is

814 evolving into a high-performance environment where coaches and players are

815 required to implement appropriate structures and models to achieve higher levels

816 of performance (Pedraza-Ramirez, 2019). This continuous evolution could be a

817 challenge in its own right from a research and applied sports psychology

818 perspective (Cottrell et al., 2018; Steinkuehler, 2019). Even though the

819 introduction of expert knowledge from traditional sports into esports is required

820 to develop sustainable performance, there have been a few cases where this

821 introduction has failed (Green, 2018). Consequently, understanding the specific

822 cultural characteristics of each esport itself is necessary to contribute to the

823 transfer of expert knowledge to esports systems. 
Running head: ESPORTS PSYCHOLOGY

\section{Acknowledgements}

826 We thank the Performance Psychology group for their critical feedback on an earlier

827 draft of this manuscript. We thank Anita Todd for English editing. Also, we would like

828 to thank the participants of the discussion session at the FEPSAC Congress in Münster

829 (July 15-20, 2019), who provided valuable remarks for the improvement of the

830 manuscript. No potential conflict of interest is reported by the authors. This work was

831 supported by the German Academic Exchange Service [DAAD] under grant [blinded

832 for peer review]. 
Running head: ESPORTS PSYCHOLOGY

\section{References}

834 References marked with an asterisk indicate studies included in this systematic review.

835 Abbott, A., \& Collins, D. (2004). Eliminating the dichotomy between theory and

836 practice in talent identification and development: Considering the role of

837 psychology. Journal of Sport Science, 22(5), 395-408.

838 https://doi.org/10.1080/02640410410001 675324

839

*Aliyari, H., Kazemi, M., Tekieh, E., Salehi, M., Sahraei, H., Daliri, M. R., ... Aghdam, A. R. (2015). The effects of Fifa 2015 computer games on changes in cognitive, hormonal and brain waves functions of young men volunteers. Basic and Clinical Neuroscience, 6(3), 193-201.

Anderson, C. A., Shibuya, A., Ihori, N., Swing, E. L., Bushman, B. J., Sakamoto, A., ... Saleem, M. (2010). Violent video game effects on aggression, empathy, and prosocial behavior in Eastern and Western countries: A meta-analytic review. Psychological Bulletin, 136(2), 151-173. https://doi.org/10.1037/a0018251

Bányai, F., Griffiths, M. D., Király, O., \& Demetrovics, Z. (2019). The psychology of esports: A systematic literature review. Journal of Gambling Studies, 35(2), 351365. https://doi.org/10.1007/s10899-018-9763-1

*Bavelier, D., Achtman, R., Mani, M., \& Föcker, J. (2012). Neural bases of selective attention in action video game players. Vision Research, 61, 132-143. https://doi.org/10.1016/j.visres.2011.08.007

853 Bediou, B., Adams, D. M., Mayer, R. E., Tipton, E., Green, C. S., \& Bavelier, D. 854 (2018). Meta-analysis of action video game impact on perceptual, attentional, and cognitive skills. Psychological Bulletin, 144(1), 77-110. https://doi.org/10.1037/bul0000130 
Running head: ESPORTS PSYCHOLOGY

858

859

860

861

862

863

864

865

866

867

868

869

870

871

872

873

874

875

876

877

878

879

880

881

882

(2014). Action video game play facilitates the development of better perceptual templates. Proceedings of the National Academy of Sciences of the United States of America, 111(47), 16961-16966. https://doi.org/10.1073/pnas.1417056111

*Bertran, E., \& Chamarro, A. (2016). Videogamers of League of Legends: The role of passion in abusive use and in performance. Adicciones, 28(1), 28-34. https://doi.org/10.20882/adicciones. 787

*Billieux, J., Van der Linden, M., Achab, S., Khazaal, Y., Paraskevopoulos, L., Zullino, D., \& Thorens, G. (2013). Why do you play World of Warcraft? An in-depth exploration of self-reported motivations to play online and in-game behaviours in the virtual world of Azeroth. Computers in Human Behavior, 29(1), 103-109. https://doi.org/10.1016/j.chb.2012.07.021

*Bonny, J., \& Castaneda, L. (2016). Impact of the arrangement of game information on recall performance of multiplayer online battle arena players. Applied Cognitive Psychology, 30(5), 664-671. https://doi.org/10.1002/acp.3234

*Bonny, J., \& Castaneda, L. (2017). Number processing ability is connected to longitudinal changes in multiplayer online battle arena skill. Computers in Human Behavior, 66, 377-387. https://doi.org/10.1016/j.chb.2016.10.005

*Boot, W. R., Kramer, A. F., Simons, D. J., Fabiani, M., \& Gratton, G. (2008). The effects of video game playing on attention, memory, and executive control. Acta Psychologica, 129(3), 387-398. https://doi.org/10.1016/j.actpsy.2008.09.005

*Bowman, N. D., Weber, R., Tamborini, R., \& Sherry, J. (2013). Facilitating game play: How others affect performance at and enjoyment of video games. Media Psychology, 16(1), 39-64. https://doi.org/10.1080/15213269.2012.742360

*Breuer, J., Scharkow, M., \& Quandt, T. (2013). Sore losers? A reexamination of the frustration-aggression hypothesis for colocated video game play. Psychology of 
Running head: ESPORTS PSYCHOLOGY

883

884

885

886

887

888

889

890

891

892

893

894

895

896

897

898

899

900

901

902

903

904

905

906

907

Popular Media Culture, 4(2), 126-137. https://doi.org/10.1037/ppm0000020

Campbell, M. J., Toth, A. J., Moran, A. P., Kowal, M., \& Exton, C. (2018). eSports: A new window on neurocognitive expertise? In S. Marcora \& M. Sarkar (Eds.), Sport and the brain: The science of preparing, enduring, and winning, part C (Progress in Brain Research, Vol. 240, pp. 161-174). Cambridge, MA: Academic Press. https://doi.org/10.1016/bs.pbr.2018.09.006

*Castaneda, L., Sidhu, M. K., Azose, J. J., \& Swanson, T. (2016). Game play differences by expertise level in Dota 2, a complex multiplayer video game. International Journal of Gaming and Computer-Media Simulations, 8(4), 1-24. https://doi.org/10.4018/IJGCMS.2016100101

*Chang, Y.-H., Liu, D.-C., Chen, Y.-Q., \& Hsieh, S. (2017). The relationship between online game experience and multitasking ability in a virtual environment. Applied Cognitive Psychology, 31(6), 653-661. https://doi.org/10.1002/acp.3368

Cote, J., Baker, J., \& Abernethy, B. (2007). Practice and play in the development of sport expertise. In G Tenenbaum \& R. Eklund (Eds.), Handbook of sport psychology (3rd ed., pp. 184-202). Hoboken, NJ: Wiley. https://doi.org/10.1002/9781118270011.ch8

Cottrell, C., McMillen, N., \& Harris, B. S. (2018). Sport psychology in a virtual world: Considerations for practitioners working in eSports. Journal of Sport Psychology in Action, 10, 73-81. https://doi.org/10.1080/21520704.2018.1518280

Council of Europe. (2001). Recommendation No. R (92) 13 REV of the committee of ministers to member states on the revised European sports charter. Retrieved from https://rm.coe.int/16804c9dbb

Dale, G., \& Green, C. S. (2017). The changing face of video games and video gamers: Future directions in the scientific study of video game play and cognitive 
Running head: ESPORTS PSYCHOLOGY

908

909

910

911

912

913

914

915

916

917

918

919

920

921

922

923

924

925

926

927

928

929

930

931

932

performance. Journal of Cognitive Enhancement, 1(3), 280-294.

https://doi.org/10.1007/s41465-017-0015-6

Diamond, A. (2013). Executive functions. Annual Review of Psychology, 64, 135-168. https://doi.org/10.1146/annurev-psych-113011-143750

*Ding, Y., Hu, X., Li, J., Ye, J., Wang, F., \& Zhang, D. (2018). What makes a champion: The behavioral and neural correlates of expertise in multiplayer online battle arena games. International Journal of Human-Computer Interaction, 34(8), 682-694. https://doi.org/10.1080/10447318.2018.1461761

Donaldson, S. (2017). Mechanics and metagame: Exploring binary expertise in League of Legends. Games and Culture, 12(5), 426-444. https://doi.org/10.1177/1555412015590063

Ericsson, A., Krampe, R., \& Tesch-Römer, C. (1993). The role of deliberate practice in the acquisition of expert performance. Psychological Review, 100(3), 363-406. https://doi.org/10.1016/j.coi.2013.02.008

Ericsson, K. A. (2019). Towards a science of the acquisition of expert performance in sports: Clarifying the differences betweem deliberate practice and other types of practice. Journal of Sports Sciences, 12, 1-18. https://doi.org/10.1080/02640414.1019.1688618

Esports or eSports? The Associated Press puts an end to the popular debate. (2017). Retrieved from https://www.dexerto.com/news/esports-esports-associated-pressputs-end-popular-debate/32233

Garcia-Naveira, Toribio, M., Molero, B., \& Suarez, A. (2018). Beneficios cognitivos, psicológicos y personales del uso de los videojuegos y esports: Una revisión. Revista de Psicología Aplicada Al Deporte y Al Ejercicio Físico, 3, 1-15. https://doi.org/10.5093/rpadef2017a1 
Running head: ESPORTS PSYCHOLOGY

933 *Glass, B. D., Maddox, W. T., \& Love, B. C. (2013). Real-time strategy game training:

934 Emergence of a cognitive flexibility trait. PLoS ONE, 8(8), 1-7.

935 https://doi.org/10.1371/journal.pone.0070350

936 *Gong, D., He, H., Ma, W., Liu, D., Huang, M., Dong, L., .. Yao, D. (2016).

937 Functional integration between salience and central executive networks: A role for

$938 \quad$ action video game experience. Neural Plasticity, 2016.

939 https://doi.org/10.1155/2016/9803165

940 Gong, D., Ma, W., Liu, T., Yan, Y., \& Yao, D. (2019). Electronic-Sports Experience

941 Related to Functional Enhancement in Central Executive and Default Mode

$942 \quad$ Areas. Neural Plasticity, 2019, 1-7. https://doi.org/10.1155/2019/1940123

943 Goodale, G. (2003). Are video games a sport? Retrieved from

944 https://www.csmonitor.com/2003/0808/p13s01-alsp.html?entryBottomStory

$945 *^{*}$ Gray, P. B., Vuong, J., Zava, D. T., \& McHale, T. S. (2018). Testing men's hormone

946 responses to playing League of Legends: No changes in testosterone, cortisol,

947 DHEA or androstenedione but decreases in aldosterone. Computers in Human

$948 \quad$ Behavior, 83, 230-234. https://doi.org/10.1016/j.chb.2018.02.004

$949 *$ Green, C. S., \& Bavelier, D. (2003). Action video game modifies visual selective

$950 \quad$ attention. Nature, 423, 534-537. https://doi.org/10.1038/nature01647

951 *Green, C. S., \& Bavelier, D. (2006). Enumeration versus multiple object tracking: The

952 case of action video game players. Cognition, 101(1), 217-245.

$953 \quad$ https://doi.org/10.1016/j.cognition.2005.10.004

$954 *$ Green, C. S., \& Bavelier, D. (2007). Action-video-game experience alters the spatial

955 resolution of vision. Psychological Science, 18(1), 88-94.

$956 \quad$ https://doi.org/10.1111/j.1467-9280.2007.01853.x

957 Green, C. S., \& Bavelier, D. (2015). Action video game training for cognitive 
Running head: ESPORTS PSYCHOLOGY

958

959

960

961

962

963

964

965

966

967

968

969

970

971

972

973

974

975

976

977

978

979

980

981

982

enhancement. Current Opinion in Behavioral Sciences, 4, 103-108.

https://doi.org/10.1016/j.cobeha.2015.04.012

Green, W. (2018). Sport management \& sport psychology for esport: Winning championships. Retrieved from https://medium.com/@MindGamesWeldon/sportmanagement-sport-psychology-for-esport-winning-championships-717491ba4609

Hallmann, K., \& Giel, T. (2018). eSports—Competitive sports or recreational activity? Sport Management Review, 21(1), 14-20. https://doi.org/10.1016/j.smr.2017.07.011

Hamari, J., \& Sjöblom, M. (2017). What is eSports and why do people watch it? Internet Research, 27(2), 211-232. https://doi.org/10.1108/IntR-04-2016-0085

Himmelstein, D., Liu, Y., \& Shapiro, J. L. (2017). An exploration of mental skills among competitive League of Legend players. International Journal of Gaming and Computer-Mediated Simulations, 9(2), 1-21.

https://doi.org/10.4018/IJGCMS.2017040101

*Hopp, T., \& Fisher, J. (2017). Examination of the relationship between gender, performance, and enjoyment of a first-person shooter game. Simulation \& Gaming, 48(3), 338-362. https://doi.org/10.1177/1046878117693397

*Huang, J., Yan, E., Cheung, G., Nagappan, N., \& Zimmermann, T. (2017). Master maker: Understanding gaming skill through practice and habit from gameplay behavior. Topics in Cognitive Science, 9(2), 437-466. https://doi.org/10.1111/tops.12251

*Hubert-Wallander, B., Green, C. S., Sugarman, M., \& Bavelier, D. (2011). Changes in search rate but not in the dynamics of exogenous attention in action videogame players. Attention, Perception and Psychophysics, 73(8), 2399-2412. https://doi.org/10.3758/s13414-011-0194-7 
Running head: ESPORTS PSYCHOLOGY

983 *Hudson, M., \& Cairns, P. (2016). The effects of winning and losing on social presence 984 in team-based digital games. Computers in Human Behavior, 60, 1-12.

985 https://doi.org/10.1016/j.chb.2016.02.001

986

*Hyun, G. J., Shin, Y. W., Kim, B.-N., Cheong, J. H., Jin, S. N., \& Han, D. H. (2013).

987 Increased cortical thickness in professional on-line gamers. Psychiatry

988 Investigation, 10(4), 388-392. https://doi.org/10.4306/pi.2013.10.4.388

989

*Kahn, A. S., \& Williams, D. (2016). We're all in this (game) together: Transactive

990 memory systems, social presence, and team structure in multiplayer online battle

991

992 arenas. Communication Research, 43(4), 487-517.

993 https://doi.org/10.1177/0093650215617504

*Kimble, C. E., \& Rezabek, J. S. (1992). Playing games before an audience: Social 994 facilitation or choking. Social Behavior and Personality: An International Journal, 995 20(2), 115-120. https://doi.org/10.2224/sbp.1992.20.2.115

996

*Klaffehn, A. L., Schwarz, K. A., Kunde, W., \& Pfister, R. (2018). Similar task997 switching performance of real-time strategy and first-person shooter players:

*Kokkinakis, A. V, Cowling, P. I., Drachen, A., \& Wade, A. R. (2017). Exploring the relationship between video game expertise and fluid intelligence. PLOS ONE, 12(11). https://doi.org/10.1371/journal.pone.0186621

1003 Korea e-Sports Association. (2014). History of Korea e-Sports Association 1999-2004.

1004 Retrieved from https://www.theverge.com/2013/9/30/4719766/twitch-raises-20million-esports-market-booming

*Kowalczyk, N., Shi, F., Magnuski, M., Skorko, M., Dobrowolski, P., Kossowski, B., ... Brzezicka, A. (2018). Real-time strategy video game experience and structural 
Running head: ESPORTS PSYCHOLOGY

1008

1009

1010

1011

1012

1013

1014

1015

1016

1017

1018

1019

1020

1021

1022

1023

1024

1025

1026

1027

1028

1029

1030

1031

1032 connectivity-A diffusion tensor imaging study. Human Brain Mapping, 39(9), 3742-3758. https://doi.org/10.1002/hbm.24208

*Lau-Zhu, A., Holmes, E. A., Butterfield, S., \& Holmes, J. (2017). Selective association between Tetris game play and visuospatial working memory: A preliminary investigation. Applied Cognitive Psychology, 31(4), 438-445. https://doi.org/10.1002/acp.3339

Lemmens, J. S., \& Hendriks, S. J. F. (2016). Addictive online games: Examining the relationship between game genres and internet gaming disorder. Cyberpsychology, Behavior, and Social Networking, 19(4), 270-276. https://doi.org/10.1089/cyber.2015.0415

Li, R. (2016). Good luck have fun: The rise of eSports. New York, NY: Skyhorse Publishing.

Liberati, A., Altman, D., Tetzlaff, J., Mulrow, C., Gøtzsche, P., Ioannidis, J., ... Moher, D. (2009). The PRISMA statement for reporting systematic reviews and metaanalyses of studies that evaluate health care interventions: Explanation and elaboration. Journal of Clinical Epidemiology, 62(10), e1-e34. https://doi.org/10.1016/j.jclinepi.2009.06.006

*Lindstedt, J. K., \& Gray, W. D. (2019). Distinguishing experts from novices by the mind's hand and mind's eye. Cognitive Psychology, 109, 1-25. https://doi.org/10.1016/j.cogpsych.2018.11.003

Maciej, B., Kosakowski, M., \& Kaczmarek, L. D. (2020). Social challenge and threat predict performance and cardiovascular responses during competitive video gaming. Psychology of Sport and Exercise, 46, 101584. https://doi.org/10.1016/j.psychsport.2019.101584

*Maglio, P. P., Wenger, M. J., \& Copeland, A. M. (2008). Evidence for the role of self- 
Running head: ESPORTS PSYCHOLOGY

1033

1034

1035

1036

1037

1038

1039

1040

1041

1042

1043

1044

1045

1046

1047

1048

1049

1050

1051

1052

1053

1054

1055

1056

1057

priming in epistemic action: Expertise and the effective use of memory. Acta Psychologica, 127(1), 72-88. https://doi.org/10.1016/j.actpsy.2007.02.001

Miyake, A., Friedman, N.P., Emerson, M.J., Witzki, A. H., Howerter, A., Wager, T. D. (2000). The unity and diversity of executive functions and their contributions to complex "frontal lobe" tasks: A latent variable analysis. Cognitive Psychology, 41(1), 49-100. https://doi.org/10.1006/cogp.1999.0734

*Momi, D., Smeralda, C., Sprugnoli, G., Ferrone, S., Rossi, S., Rossi, A., ... Santarnecchi, E. (2018). Acute and long-lasting cortical thickness changes following intensive first-person action videogame practice. Behavioural Brain Research, 353, 62-73. https://doi.org/10.1016/j.bbr.2018.06.013

Moola, S., Munn, Z., Tufanaru, C., Aromataris, E., Sears, K., Sfetcu, R., ... Mu, P. (2017). Systematic reviews of etiology and risk. In E. Aromataris \& Z. Munn (Eds.), Joanna Briggs Institute reviewer's manual. Retrieved from https://reviewersmanual.joannabriggs.org/

Mora-Cantallops, M., \& Sicilia, M.-Á. (2018). MOBA games: A literature review. Entertainment Computing, 26, 128-138. https://doi.org/10.1016/j.entcom.2018.02.005

Murphy, S. (2009). Video games, competition and exercise: A new opportunity for sport psychologists? The Sport Psychologist, 23(4), 487-503. https://doi.org/10.1123/tsp.23.4.487

Musculus, L. (2018). Do the best players "take-the-first”? Examining expertise differences in the option-generation and selection processes of young soccer players. Sport, Exercise, and Performance Psychology, 7(3), 271-283. https://doi.org/10.1037/spy0000123

Musculus, L., Ruggeri, A., Raab, M., \& Lobinger, B. (2019). A developmental 
Running head: ESPORTS PSYCHOLOGY

1058

1059

1060

1061

1062

1063

1064

1065

1066

1067

1068

1069

1070

1071

1072

1073

1074

1075

1076

1077

1078

1079

1080

1081

1082

perspective on option generation and selection. Developmental Psychology, 55, 45-753. https://doi.org/10.1037/dev0000665

Nitsch, J. R., \& Hackfort, D. (2015). Theoretical framework of performance psychology. In M. Raab, B. Lobinger, S. Hoffmann, A. Pizzera, \& S. Laborde (Eds.), Performance psychology: Perception, action, cognition, and emotion (pp. 11-29). London, England: Academic Press. https://doi.org/10.1016/b978-0-12$803377-7.00002-8$

*Okagaki, L., \& Frensch, P. A. (1994). Effects of video game playing on measures of spatial performance: Gender effects in late adolescence. Journal of Applied Developmental Psychology, 15, 33-58. https://doi.org/10.1016/01933973(94)90005-1

*Oxford, J., Ponzi, D., \& Geary, D. C. (2010). Hormonal responses differ when playing violent video games against an ingroup and outgroup. Evolution and Human Behavior, 31(3), 201-209. https://doi.org/10.1016/j.evolhumbehav.2009.07.002

Parshakov, P., Coates, D., \& Zavertiaeva, M. (2018). Is diversity good or bad? Evidence from eSports teams analysis. Applied Economics, 50(47), 5062-5073. https://doi.org/10.1080/00036846.2018.1470315

Pedraza-Ramirez, I. (2019). Generación LoL: Entrenamiento psicológico mediante una propuesta holística con un equipo profesional de esports. Revista de Psicología Aplicada Al Deporte y El Ejercicio Físico, 4(1), 1-10. https://doi.org/10.5093/rpadef2019a3

*Pereira, R., Wilwert, M. L., \& Takase, E. (2016). Contributions of sport psychology to the competitive gaming: An experience report with a professional team of League of Legends. International Journal of Applied Psychology, 6(2), 27-30. https://doi.org/10.5923/j.ijap.20160602.01 
Running head: ESPORTS PSYCHOLOGY

1083 Petticrew, M., \& Roberts, H. (2008). Systematic reviews-Do they 'work' in informing 1084 decision-making around health inequalities? Health Economics, Policy and Law, 3(2), 197-211. https://doi.org/10.1017/s1744133108004453

1086

*Pilegard, C., \& Mayer, R. E. (2018). Game over for Tetris as a platform for cognitive skill training. Contemporary Educational Psychology, 54, 29-41. https://doi.org/10.1016/j.cedpsych.2018.04.003

*Pirker, J., Rattinger, A., Drachen, A., \& Sifa, R. (2018). Analyzing player networks in Destiny. Entertainment Computing, 25, 71-83.

1091 https://doi.org/10.1016/j.entcom.2017.12.001

Pluss, M. A., Bennett, K. J. M., Novak, A. R., Panchuk, D., Coutts, A. J., \& Fransen, J. (2019). Esports: The chess of the 21st century. Frontiers in Psychology, 10. https://doi.org/10.3389/fpsyg.2019.00156

Popper, B. (2013). Field of streams: How Twitch made video games a spectator sport. Retrieved from https://www.theverge.com/2013/9/30/4719766/twitch-raises-20million-esports-market-booming

Powers, K. L., Brooks, P. J., Aldrich, N. J., Palladino, M. A., \& Alfieri, L. (2013). Effects of video-game play on information processing: A meta-analytic investigation. Psychonomic Bulletin and Review, 20(6), 1055-1079. https://doi.org/10.3758/s13423-013-0418-z

*Qiu, N., Ma, W., Fan, X., Zhang, Y., Li, Y., Yan, Y., ... Yao, D. (2018). Rapid

1103 improvement in visual selective attention related to action video gaming experience. Frontiers in Human Neuroscience, 12. https://doi.org/10.3389/fnhum.2018.00047 psychology: Perception, action, cognition, and emotion. London, England: 
Running head: ESPORTS PSYCHOLOGY

1108

1109

1110

1111

1112

1113

1114

1115

1116

1117

1118

1119

1120

1121

1122

1123

1124

1125

1126

1127

1128

1129

1130

1131

1132

Academic Press.

Raab, M., Masters, R. S. W., \& Maxwell, J. P. (2005). Improving the "how" and "what" decisions of elite table tennis players. Human Movement Science, 24(3), 326-344. https://doi.org/10.1016/j.humov.2005.06.004

Reeves, S., Brown, B., \& Laurier, E. (2009). Experts at play: Understanding skilled expertise. Games and Culture, 4(3), 205-227. https://doi.org/10.1177/1555412009339730

*Röhlcke, S., Bäcklund, C., Sörman, D. E., \& Jonsson, B. (2018). Time on task matters most in video game expertise. PLoS ONE, 13(10). https://doi.org/10.1371/journal.pone.0206555

Ruvalcaba, O., Shulze, J., Kim, A., Berzenski, S. R., \& Otten, M. P. (2018). Women's experiences in eSports: Gendered differences in peer and spectator feedback during competitive video game play. Journal of Sport \& Social Issues, 42(4), 295-311. https://doi.org/10.1177/0193723518773287

Sala, G., Tatlidil, K. S., \& Gobet, F. (2017). Video game training does not enhance cognitive ability: A comprehensive meta-analytic investigation. Psychological Bulletin, 144(2), 111-139. https://doi.org/10.1037/bu10000139

*Sapienza, A., Zeng, Y., Bessi, A., Lerman, K., \& Ferrara, E. (2018). Individual performance in team-based online games. Royal Society Open Science, 5(6). https://doi.org/10.1098/rsos.180329

Schmidt, R., Lee, T., Winstein, C., Wulf, G., \& Zelaznik, H. (1999). Motor control and learning: A behavioral emphasis (6th ed.). Champaign, IL: Human Kinetics.

Schwarz, A. M., Schächinger, H., Adler, R. H., \& Goetz, S. M. (2003). Hopelessness is associated with decreased heart rate variability during championship chess games. Psychosomatic Medicine, 65(4), 658-661. 
Running head: ESPORTS PSYCHOLOGY

1133

1134

1135

1136

1137

1138

1139

1140

1141

1142

1143

1144

1145

1146

1147

1148

1149

1150

1151

1152

1153

1154

1155

1156

1157

https://doi.org/10.1097/01.PSY.0000075975.90979.2A

*Seya, Y., \& Shinoda, H. (2016). Experience and training of a first person shooter (FPS) game can enhance useful field of view, working memory, and reaction time. International Journal of Affective Engineering, 15(3), 213-222. https://doi.org/10.5057/ijae.ijae-d-15-00014

Shamseer, L., Moher, D., Clarke, M., Ghersi, D., Liberati, A., Petticrew, M., ... Whitlock, E. (2015). Preferred reporting items for systematic review and metaanalysis protocols (PRISMA-P) 2015 statement. British Medical Journal, 20(2), 148-160. https://doi.org/10.1186/2046-4053-4-1

*Sibert, C., Gray, W. D., \& Lindstedt, J. K. (2017). Interrogating feature learning models to discover insights into the development of human expertise in a real-time, dynamic decision-making task. Topics in Cognitive Science, 9(2), 374-394. https://doi.org/10.1111/tops.12225

Starkes, J., \& Ericsson, K. A. (2003). Expert Performance in Sports: Advances in research on sport expertise. Champaign, IL, United States: Human Kinetics.

Steinkuehler, C. (2019). Esports research: Critical, empirical, and historical studies of competitive videogame play. Games and Culture. https://doi.org/10.1177/1555412019836855

*Tafalla, R. J. (2007). Gender differences in cardiovascular reactivity and game performance related to sensory modality in violent video game play. Journal of Applied Social Psychology, 37(9), 2008-2023. https://doi.org/10.1111/j.15591816.2007.00248.x

*Tanaka, S., Ikeda, H., Kasahara, K., Kato, R., Tsubomi, H., Sugawara, S. K., ... Watanabe, K. (2013). Larger right posterior parietal volume in action video game experts: A behavioral and voxel-based morphometry (VBM) study. PLoS ONE, 
Running head: ESPORTS PSYCHOLOGY

1158

1159

1160

1161

1162

1163

1164

1165

1166

1167

1168

1169

1170

1171

1172

1173

1174

1175

1176

1177

1178

1179

1180

1181

1182

8(6). https://doi.org/10.1371/journal.pone.0066998

Tang, W.Y. (2018). Understanding esports from the perspective of team dynamics. The Sport Journal. Retrieved from http://thesportjournal.org/article/understandingesports-from-the-perspective-of-team-dynamics/

Tassi, P. (2012, December 20). 2012: The year of eSports. Forbes. Retrieved from https://www.forbes.com/sites/insertcoin/2012/12/20/2012-the-year-ofesports/\#2174fa227e11

*Tekofsky, S., Spronck, P., Goudbeek, M., Plaat, A., \& van den Herik, J. (2015). Past our prime: A study of age and play style development in Battlefield 3. IEEE Transactions on Computational Intelligence and AI in Games, 7(3), 292-303. https://doi.org/10.1109/tciaig.2015.2393433

Tenenbaum, G., \& Filho, E. (2016). Measurement considerations in performance psychology. In M. Raab, B. Lobinger, S. Hoffmann, A. Pizzera, \& S. Laborde (Eds.), Performance psychology: Perception, action, cognition, and emotion (pp. 31-44). London, England: Academic Press. https://doi.org/10.1016/B978-0-12803377-7.00003-X

*Terlecki, M., Newcombe, N. S., \& Little, M. (2008). Durable and generalized effects of spatial experience on mental rotation: Gender differences in growth patterns. Applied Cognitive Psychology, 22, 996-1013. https://doi.org/10.1002/acp.1420

*Thompson, J. J., Blair, M. R., Chen, L., \& Henrey, A. J. (2013). Video game telemetry as a critical tool in the study of complex skill learning. PLoS ONE, 8(9). https://doi.org/10.1371/journal.pone.0075129

*Thompson, J. J., Blair, M. R., \& Henrey, A. J. (2014). Over the hill at 24: Persistent age-related cognitive-motor decline in reaction times in an ecologically valid video game task begins in early adulthood. PLoS ONE, 9(4). 
Running head: ESPORTS PSYCHOLOGY

1183

1184

1185

1186

1187

1188

1189

1190

1191

1192

1193

1194

1195

1196

1197

1198

1199

1200

1201

1202

1203

1204

1205

1206

1207

https://doi.org/10.1371/journal.pone.0094215

*Thompson, J. J., McColeman, C. M., Stepanova, E. R., \& Blair, M. R. (2017). Using video game telemetry data to research motor chunking, action latencies, and complex cognitive-motor skill learning. Topics in Cognitive Science, 9(2), 467484. https://doi.org/10.1111/tops. 12254

Thompson, J. J., Mccoleman, C. M., Blair, M. R., \& Henrey, A. J. (2019). Classic motor chunking theory fails to account for behavioural diversity and speed in a complex naturalistic task. Plos One, 14(6), 1-24. https://doi.org/10.1371/journal.pone.0218251

Troubat, N., Fargeas-Gluck, M. A., Tulppo, M., \& Dugué, B. (2009). The stress of chess players as a model to study the effects of psychological stimuli on physiological responses: An example of substrate oxidation and heart rate variability in man. European Journal of Applied Physiology, 105(3), 343-349. https://doi.org/10.1007/s00421-008-0908-2

Voss, M., Kramer, A., Basak, C., Prakash, R., \& Roberts, B. (2010). Are expert athletes 'expert' in the cognitive laboratory? A meta-analytic review of cognition and sport expertise. Applied Cognitive Psychology, 24, 812-826. https://doi.org/10.1002/acp

Walton, C. C., Keegan, R. J., Martin, M., \& Hallock, H. (2018). The potential role for cognitive training in sport: More research needed. Frontiers in Psychology, 9. https://doi.org/10.3389/fpsyg.2018.01121

*Wang, H., Yang, H.-T., \& Sun, C.-T. (2015). Thinking style and team competition game performance and enjoyment. IEEE Transactions on Computational Intelligence and AI in Games, 7(3), 243-254. https://doi.org/10.1109/TCIAIG.2015.2466240

Wang, P., Liu, H., Zhu, X., Meng, T., Li, H., \& Zuo, X. (2016). Action video game 
Running head: ESPORTS PSYCHOLOGY

1208

1209

1210 Witkowski, E. (2012). On the digital playing field. Games and Culture, 7(5), 349-374.

$1211 \quad$ https://doi.org/10.1177/1555412012454222

1212 Wulf, G., \& Lewthwaite, R. (2016). Optimizing performance through intrinsic motivation and attention for learning: The OPTIMAL theory of motor learning. Psychonomic Bulletin and Review, 23(5), 1382-1414. https://doi.org/10.3758/s13423-015-0999-9

*Xia, B., Wang, H., \& Zhou, R. (2017). What contributes to success in MOBA games? An empirical study of Defense of the Ancients 2. Games and Culture, 1-25. https://doi.org/10.1177/1555412017710599

Yee, N. (2006). The demographics, motivations and derived experiences of users of massively multi-user online graphical environments. Presence: Teleoperators and Virtual Environments, 15(3), 309-329. https://doi.org/doi:10.1162/pres.15.3.309 
Running head: ESPORTS PSYCHOLOGY

1224 Figure captions

1225 Figure 1. Flow diagram of the article-identification process following the Preferred

1226 Reporting Items for Systematic Review and Meta-Analysis Protocols.

1227

1228 Figure 2. Heuristic model of esports performance, depicting the bidirectional influence

1229 of esports cognitive and in-game factors of performance. All constructs could be

1230 connected but are not display in the figure.

1231

1232

1233

1234

1235

1236

1237

1238

1239

1240

1241

1242

1243

1244

1245

1246

1247

1248 


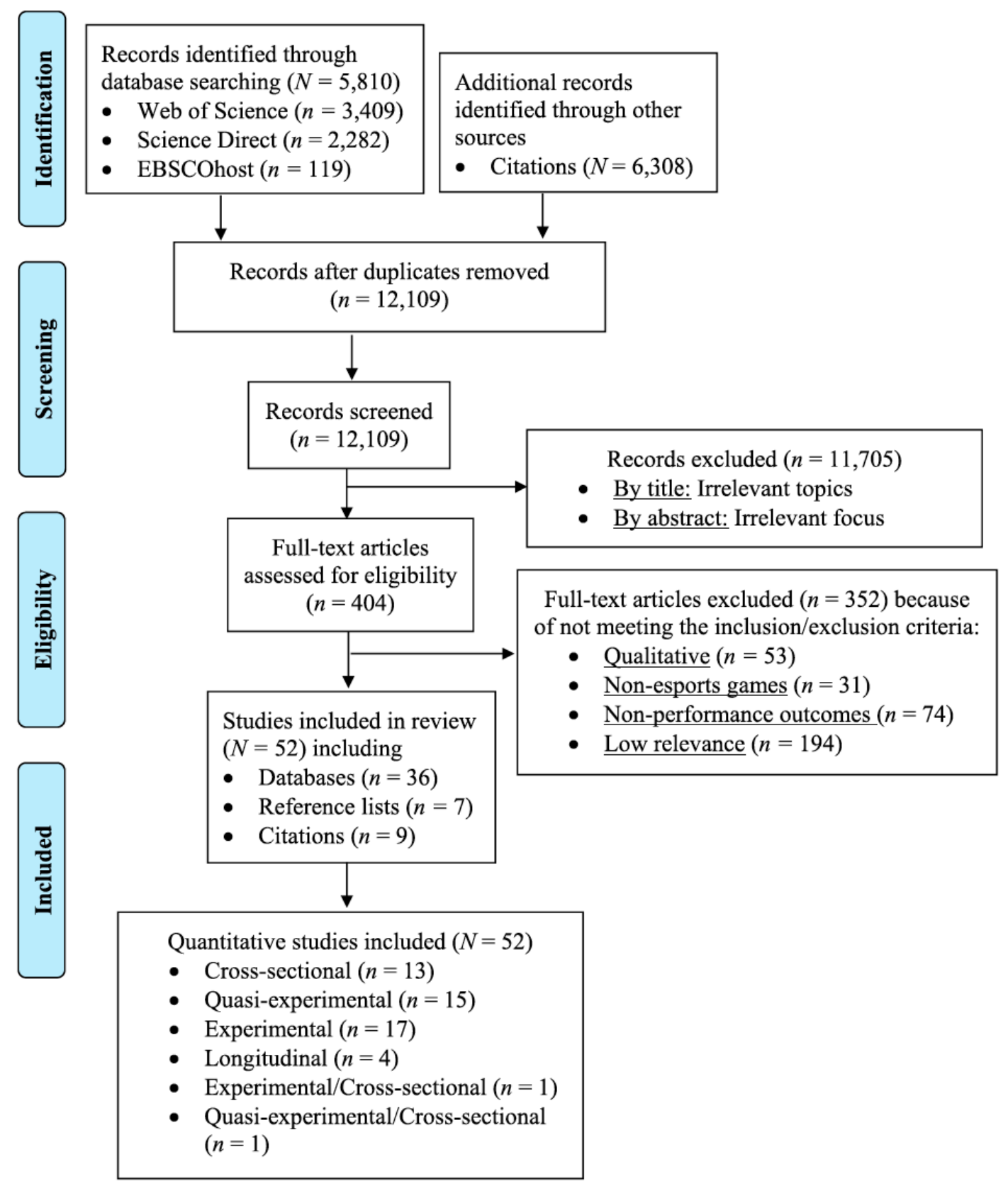

Figure 1. Flow diagram of the article-identification process following the preferred reporting items for systematic review and meta-analysis protocols. 


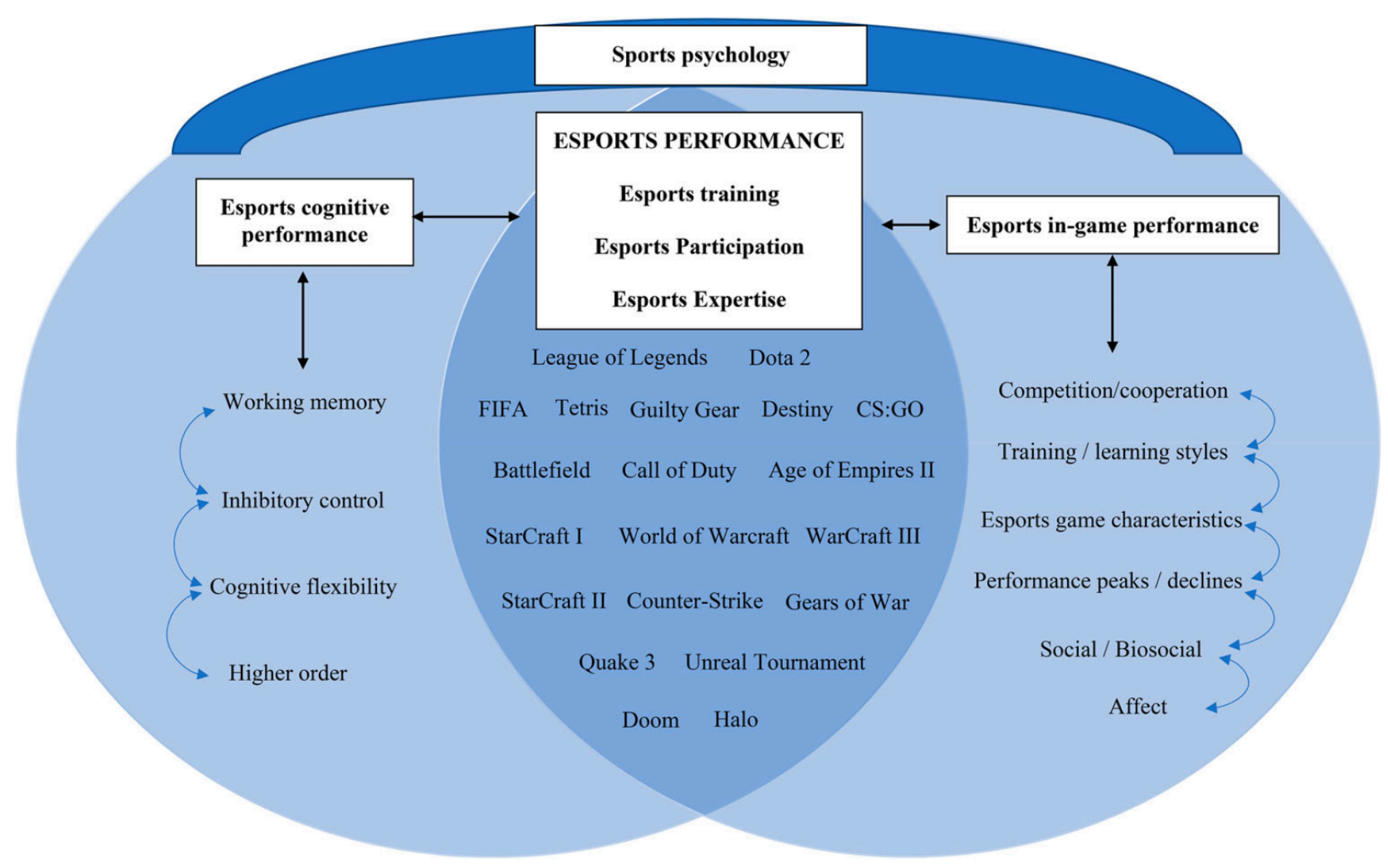

Figure 2. Heuristic model of esports performance, depicting the bidirectional influence of esports cognitive and in-game factors of performance. 
Table 1. Esports games

\begin{tabular}{|c|c|c|c|c|}
\hline Game genre ${ }^{a}$ & Esports game $^{b}$ & Release date & Tournaments ${ }^{c}$ & Participating players ${ }^{d}$ \\
\hline \multirow[t]{7}{*}{ Fighting games } & Super Street Fighter Series & 1994 & 255 & 297 \\
\hline & Guilty Gear Series* & 1998 & 77 & 262 \\
\hline & Street Fighter Series & 1999 & 120 & 518 \\
\hline & Super Smash Bros. Series & 1999 & 3701 & 3452 \\
\hline & Marvel vs. Capcom Series & 2000 & 41 & 108 \\
\hline & Tekken Series & 2002 & 227 & 350 \\
\hline & Killer Instinct & 2013 & 43 & 120 \\
\hline \multirow{5}{*}{$\begin{array}{l}\text { Real-time strategy (RTS) } \\
\text { games }\end{array}$} & Age of Empires Series* & 1997 & 205 & 549 \\
\hline & StarCraft: Brood War & 1998 & 536 & 642 \\
\hline & WarCraft III * & 2002 & 1286 & 563 \\
\hline & $\begin{array}{l}\text { World of WarCraft } \\
\text { (WoW)* }\end{array}$ & 2004 & 115 & 406 \\
\hline & StarCraft II* & 2010 & 5308 & 1895 \\
\hline \multirow{9}{*}{$\begin{array}{l}\text { Shooters: First-person } \\
\text { shooters (FPSs) and third- } \\
\text { person shooters (TPSs) }\end{array}$} & Doom Series* & 1994 & 7 & 21 \\
\hline & Quake Series* & 1996 & 769 & 829 \\
\hline & Unreal Tournament Series* & 1999 & 39 & 118 \\
\hline & Call of Duty Series (CoD)* & 2003 & 930 & 2919 \\
\hline & Halo Series* & 2004 & 284 & 887 \\
\hline & Painkiller & 2004 & 13 & 48 \\
\hline & Battlefield Series* & 2006 & 169 & 224 \\
\hline & Rainbow Six: Vegas & 2006 & 7 & 137 \\
\hline & Counter-Strike (CS)* & 2000 & 894 & 3677 \\
\hline
\end{tabular}




\begin{tabular}{|c|c|c|c|c|}
\hline Game genre ${ }^{a}$ & Esports game $^{b}$ & Release date & Tournaments ${ }^{c}$ & Participating players ${ }^{d}$ \\
\hline & $\begin{array}{l}\text { Counter-Strike Series } \\
\text { Global Offensive } \\
(\mathrm{CS}: \mathrm{GO})^{*}\end{array}$ & 2012 & 3870 & 11119 \\
\hline & Gears of War Series* & 2006 & 34 & 182 \\
\hline & CrossFire & 2007 & 292 & 600 \\
\hline & Team Fortress 2 & 2007 & 131 & 795 \\
\hline & Rainbow Six: Siege & 2015 & 58 & 435 \\
\hline & Overwatch & 2016 & 631 & 2925 \\
\hline & Fortnite & 2017 & 173 & 1426 \\
\hline & $\begin{array}{l}\text { PlayerUnknown's } \\
\text { Battlegrounds (PUBG) }\end{array}$ & 2017 & 116 & 1319 \\
\hline & Apex Legends & 2019 & 6 & 72 \\
\hline \multirow{3}{*}{$\begin{array}{l}\text { Multiplayer online battle } \\
\text { arenas (MOBAs) }\end{array}$} & League of Legends (LoL)* & 2009 & 2208 & 6125 \\
\hline & $\begin{array}{l}\text { Defense of the Ancients } \\
\text { (Dota 2)* }\end{array}$ & 2013 & 1062 & 2859 \\
\hline & Smite & 2014 & 94 & 513 \\
\hline \multirow[t]{5}{*}{ Sports games } & FIFA Series* & 1999 & 879 & 1399 \\
\hline & Madden NFL Series & 2003 & 15 & 114 \\
\hline & $\begin{array}{l}\text { Pro Evolution Soccer } \\
\text { Series }\end{array}$ & 2003 & 36 & 102 \\
\hline & Rocket League & 2015 & 259 & 502 \\
\hline & NBA $2 \mathrm{~K}$ & 2017 & 3 & 78 \\
\hline \multirow[t]{4}{*}{ Racing } & TrackMania & 2006 & 146 & 196 \\
\hline & iRacing & 2008 & 45 & 317 \\
\hline & Project CARS & 2015 & 8 & 19 \\
\hline & $\begin{array}{l}\text { F1 esports Series } \\
\text { (simulation racing) }\end{array}$ & 2017 & 2 & 44 \\
\hline
\end{tabular}




\begin{tabular}{|c|c|c|c|c|}
\hline Game genre ${ }^{a}$ & Esports game ${ }^{b}$ & Release date & Tournaments $^{c}$ & Participating players ${ }^{d}$ \\
\hline & NASCAR Heat 3 & 2018 & 1 & 32 \\
\hline \multirow[t]{4}{*}{ Mobile } & Vainglory & 2014 & 35 & 247 \\
\hline & Arena of Valor & 2015 & 24 & 260 \\
\hline & Clash Royale & 2016 & 27 & 133 \\
\hline & $\begin{array}{l}\text { PlayerUnknown's } \\
\text { Battlegrounds (PUBG) }\end{array}$ & 2017 & 6 & 90 \\
\hline \multirow[t]{2}{*}{ Other } & Tetris* & 1989 & 7 & 232 \\
\hline & Hearthstone & 2014 & 821 & 2116 \\
\hline
\end{tabular}

1257 Notes. ${ }^{a}$ Authors doing action video game research usually combine games from the RTS, FPS, and MOBA genres. ${ }^{\mathrm{b}}$ Games with an asterisk were

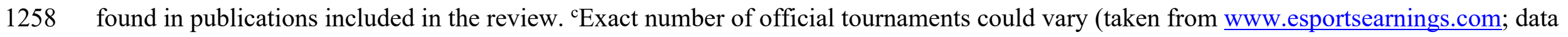

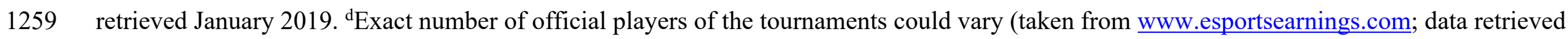

1260 January 2019.

1261

1262

1263

1264

1265

1266 
Table 2. PICO model of the systematic review

\begin{tabular}{|l|l|l|l|}
\hline Population & Intervention/Phenomena & Comparators & Outcomes \\
\hline $\begin{array}{l}\text { Esports games, general healthy } \\
\text { human population research, } \\
\text { excluding older adult population } \\
\text { and machine modelling or } \\
\text { artificial intelligence }\end{array}$ & $\begin{array}{l}\text { Psychological aspects of } \\
\text { cognitive and game } \\
\text { performance. We included } \\
\text { papers that exclusively } \\
\text { tested esports games. }\end{array}$ & $\begin{array}{l}\text { (1) Expertise level } \\
\text { (2) Differences between players } \\
\text { and non-players in cognitive and } \\
\text { game performance } \\
\text { (3) Differences between games } \\
\text { in cognitive and game } \\
\text { performance. }\end{array}$ & $\begin{array}{l}\text { Esports performance: } \\
\text { (a) Cognitive performance: participants' } \\
\text { behaviour from measures of cognitive } \\
\text { processes in a laboratory setting associated } \\
\text { with esports games. } \\
\text { (b) Game performance: winning or losing, } \\
\text { placement in a ranking system, points } \\
\text { scored, KDA, gold acquired per minute, etc. }\end{array}$ \\
\hline
\end{tabular}


Table 3. Cognitive performance

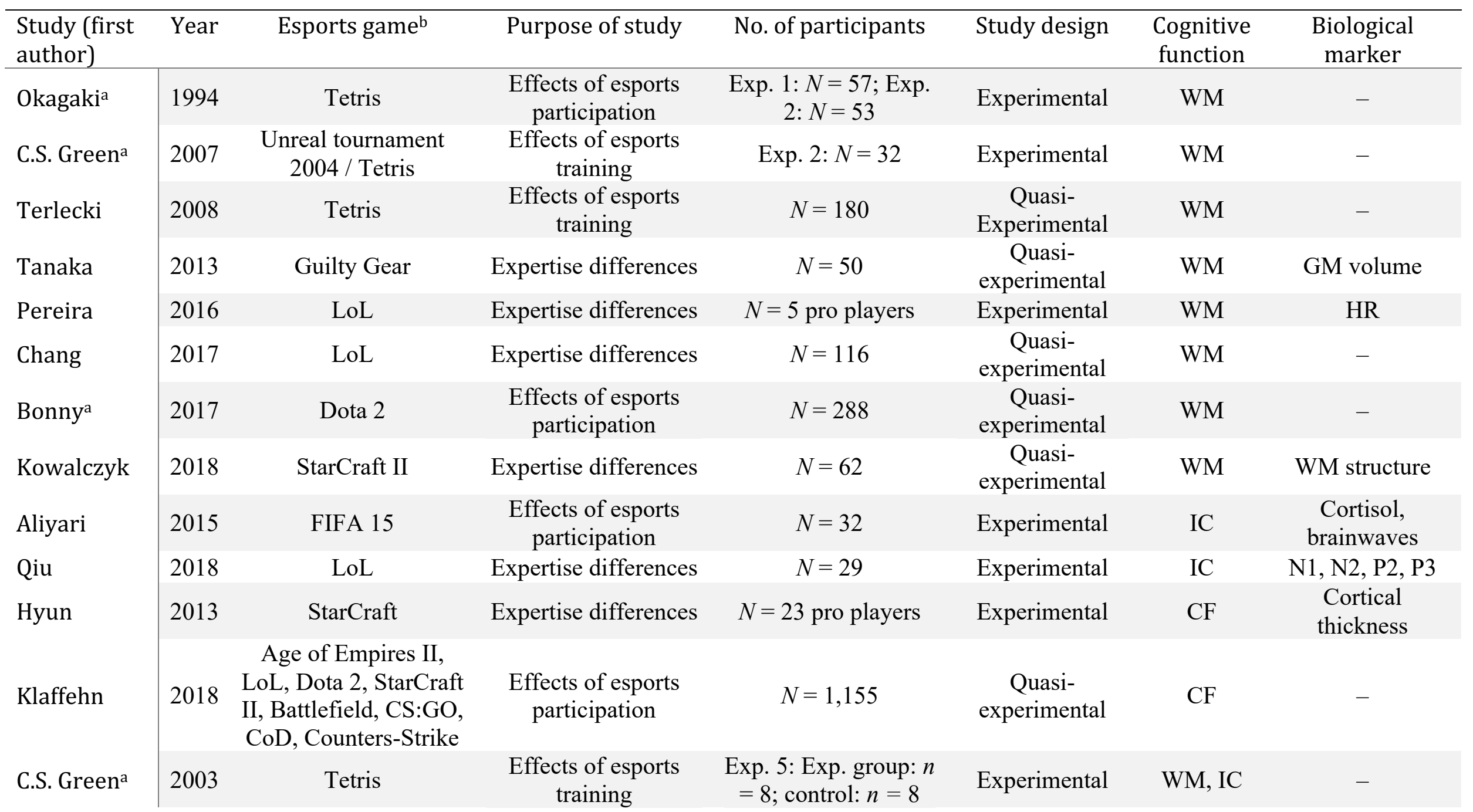




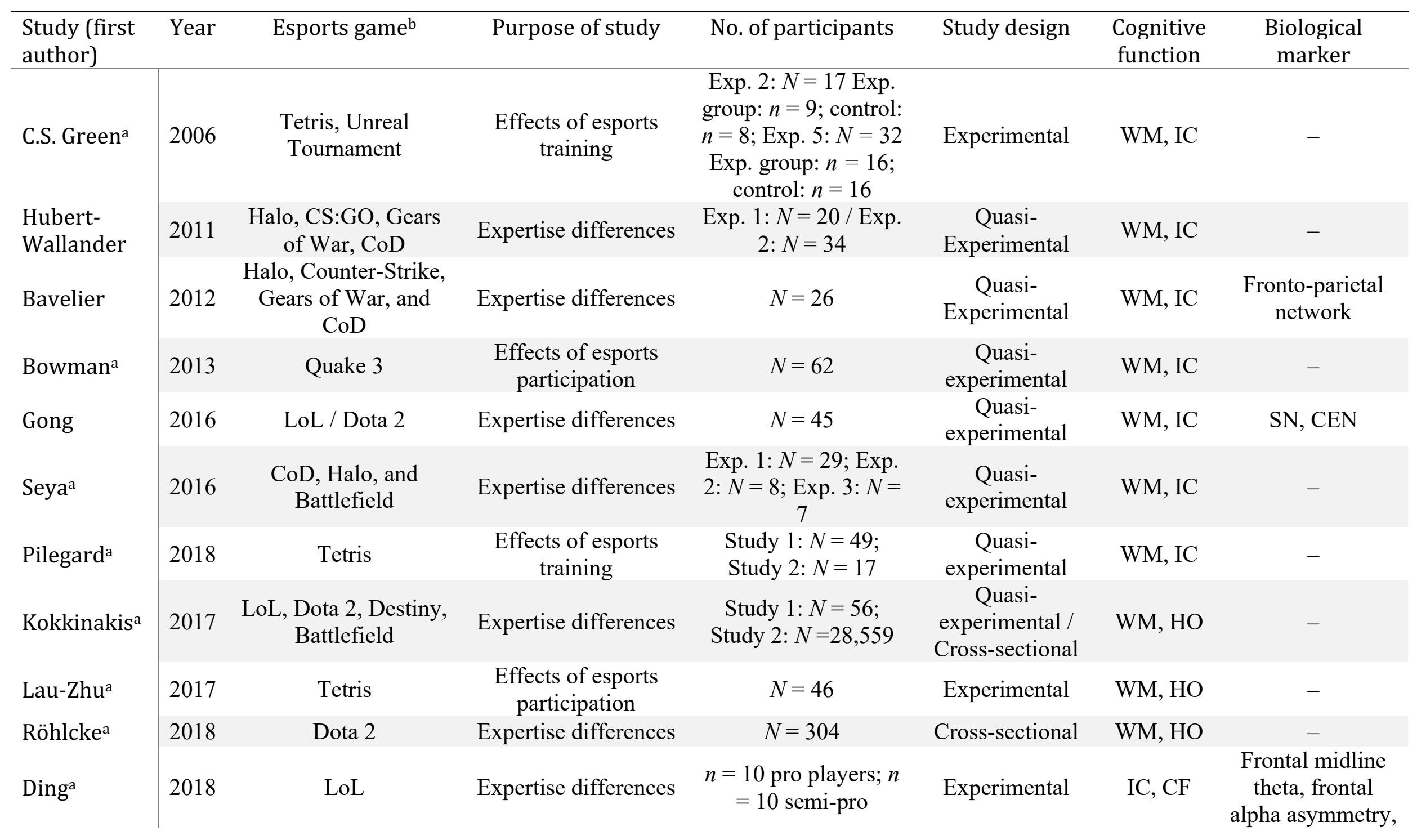




\begin{tabular}{l|cccccc}
\hline $\begin{array}{l}\text { Study (first } \\
\text { author) }\end{array}$ & Year & Esports game & Purpose of study & $\begin{array}{c}\text { No. of participants } \\
\text { trainees; } n=20 \\
\text { students }\end{array}$ & $\begin{array}{c}\text { Study design } \\
\text { Cognitive } \\
\text { function }\end{array}$ & $\begin{array}{c}\text { Biological } \\
\text { marker }\end{array}$ \\
occipital alpha, \\
HR, HRV, \\
respiration rate \\
Bejjanki
\end{tabular}

Note. Cognitive functions: $\mathrm{CF}=$ cognitive flexibility; $\mathrm{HO}=$ higher order; $\mathrm{IC}=$ inhibitory control; $\mathrm{WM}=$ working memory. Biological markers : $\mathrm{GM}$ volume = grey matter volume; $\mathrm{HR}=$ heart rate; $\mathrm{HRV}=$ heart rate variability; N1, N2, P2, P3 = event-related potentials; $\mathrm{SN}=$ salience network; $\mathrm{CEN}=$ central executive network; $\mathrm{WM}$ structure $=$ white matter structure.

a Studies selected in both categories: cognitive and game performance.

${ }^{\mathrm{b}}$ Full names of all games discussed in this review can be found in Table 1. 
Table 4. Game performance

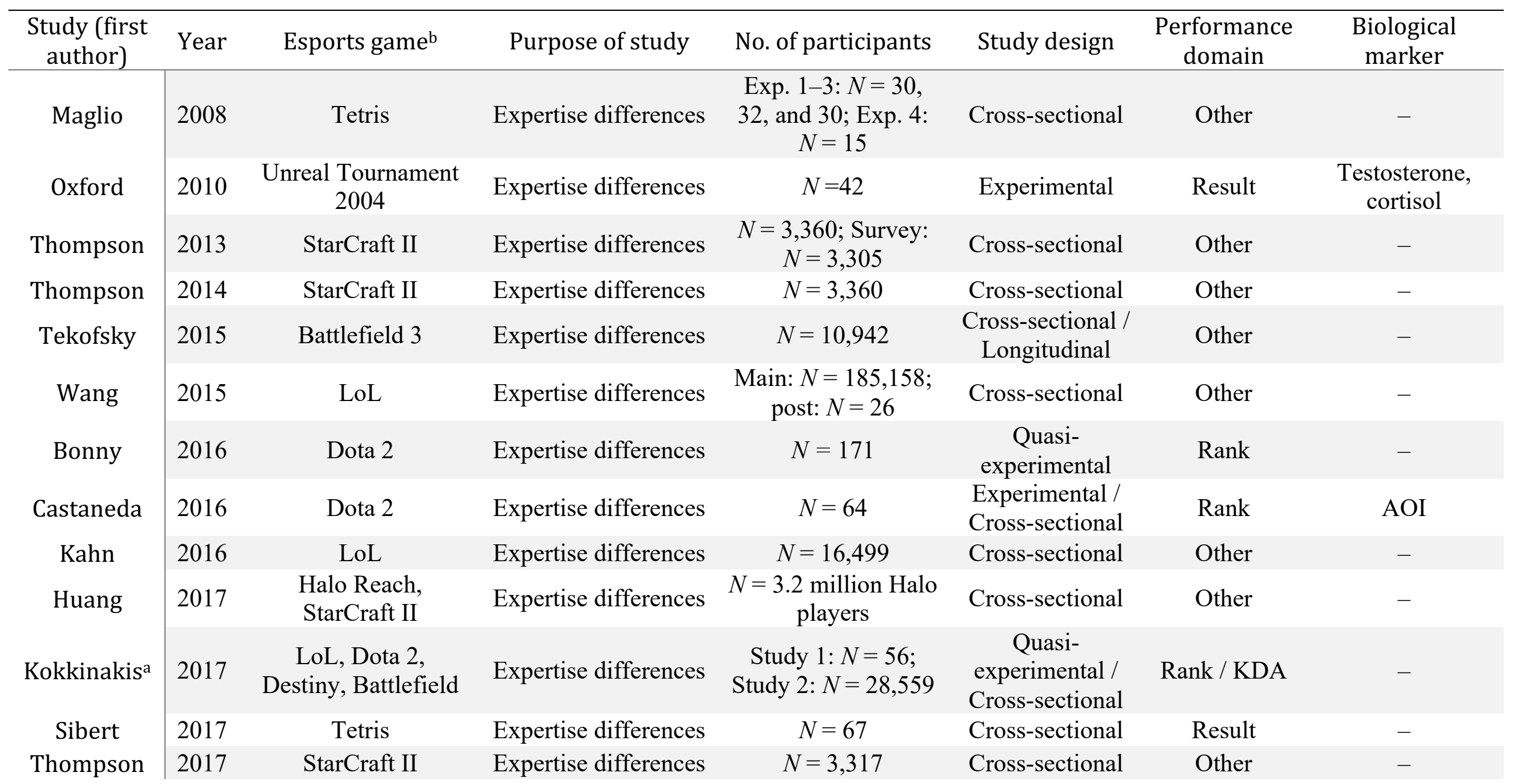




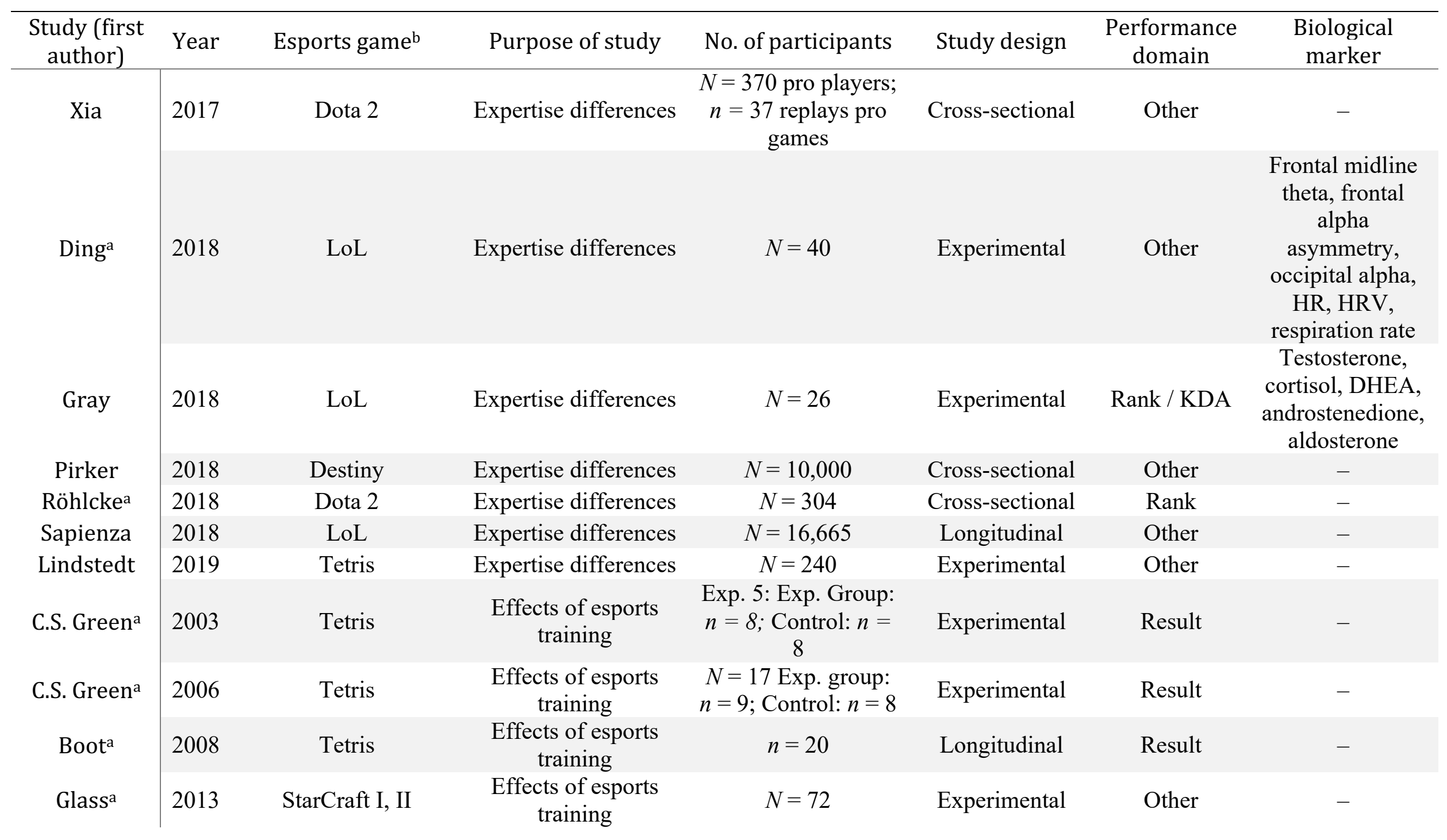




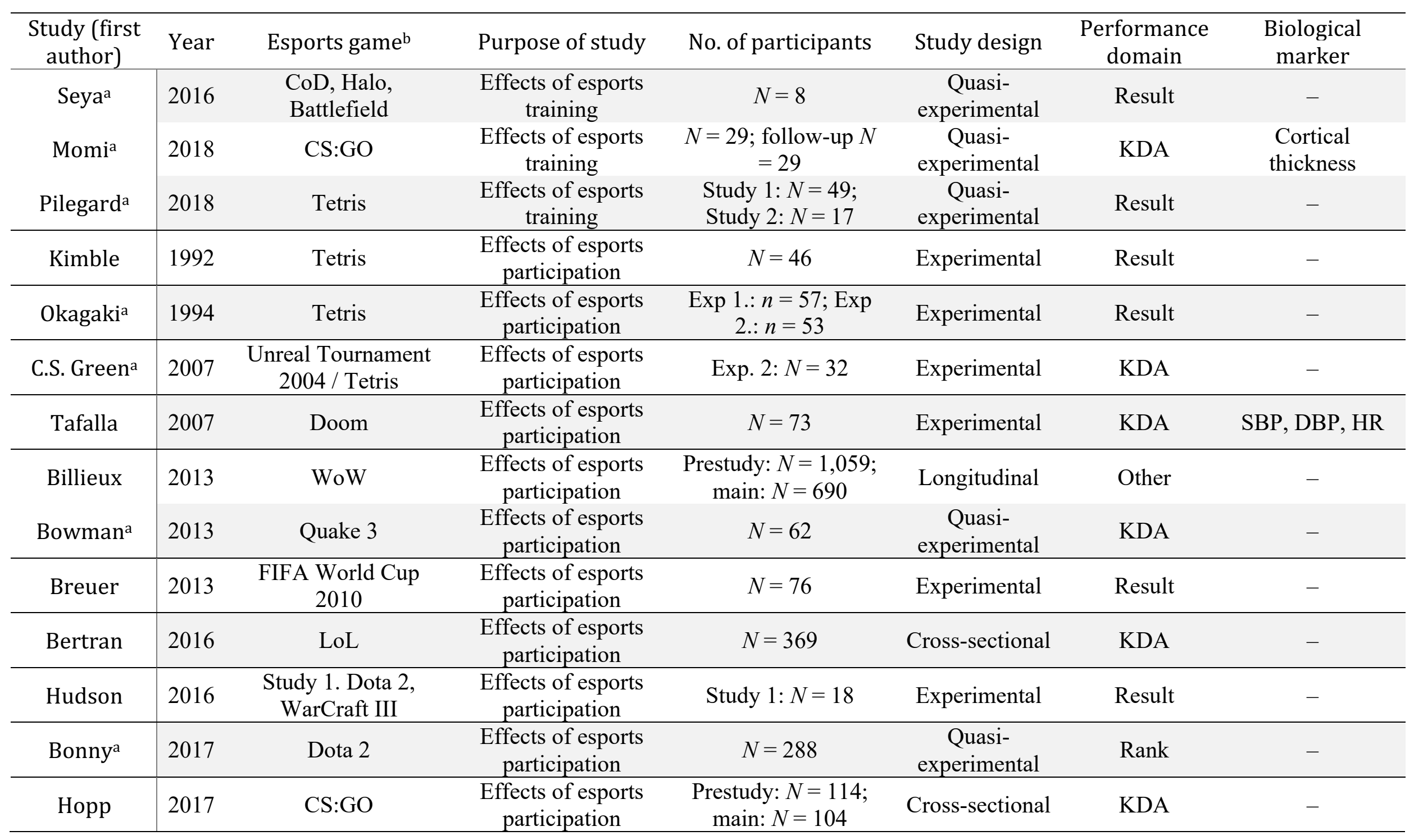




\begin{tabular}{c|ccccccc}
\hline $\begin{array}{c}\text { Study (first } \\
\text { author) }\end{array}$ & Year & Esports game $^{\mathrm{b}}$ & Purpose of study & No. of participants & Study design & $\begin{array}{c}\text { Performance } \\
\text { domain }\end{array}$ & $\begin{array}{c}\text { Biological } \\
\text { marker }\end{array}$ \\
\hline Lau-Zhu $^{\mathrm{a}}$ & 2017 & Tetris & $\begin{array}{c}\text { Effects of esports } \\
\text { participation }\end{array}$ & $N=46$ & Experimental & Result & - \\
\hline
\end{tabular}
deaths, assists; SPB = systolic blood pressure; Other = in-game measures (e.g., hotkey usage, last hitting, earned/spent gold).

${ }^{a}$ Studies selected in both categories: cognitive and game performance.

${ }^{b}$ Full names of all games discussed in this review can be found in Table 1. 